\title{
Laser-assisted nuclear decay spectroscopy of ${ }^{176,177,179} \mathrm{Au}$
}

R. D. Harding, ${ }^{1,2}$ A. N. Andreyev $\odot,{ }^{1,3,{ }^{*}}$ A. E. Barzakh, ${ }^{4}$ J. G. Cubiss, ${ }^{1}$ P. Van Duppen,,${ }^{5}$ M. Al Monthery, ${ }^{1}$ N. A. Althubiti, ${ }^{6,7}$ B. Andel,,${ }^{5,8}$ S. Antalic, ${ }^{8}$ T. E. Cocolios, ${ }^{5,7}$ T. Day Goodacre, ${ }^{2,7, \dagger}$ K. Dockx,${ }^{5}$ G. J. Farooq-Smith,${ }^{5,7}$ D. V. Fedorov, ${ }^{4}$ V. N. Fedosseev, ${ }^{2}$ D. A. Fink,${ }^{2,9}$ L. P. Gaffney,${ }^{2,10}$ L. Ghys,${ }^{5}+$ J. D. Johnson, ${ }^{5}$ D. T. Joss,${ }^{10}$ M. Huyse,${ }^{5}$ N. Imai,${ }^{11}$ K. M. Lynch, ${ }^{2,7}$ B. A. Marsh, ${ }^{2}$ Y. Martinez Palenzuela, ${ }^{2,5}$ P. L. Molkanov, ${ }^{4}$ G. G. O’Neill, ${ }^{10,}$ R. D. Page, ${ }^{10}$ R. E. Rossel, ${ }^{2,12}$ S. Rothe, ${ }^{2,12}$ M. D. Seliverstov, ${ }^{4}$ S. Sels, ${ }^{5}$ C. Van Beveren, ${ }^{5}$ and E. Verstraelen ${ }^{5}$

${ }^{1}$ Department of Physics, University of York, York, YO10 5DD, United Kingdom

${ }^{2}$ CERN, 1211, Geneva 23, Switzerland

${ }^{3}$ Advanced Science Research Center (ASRC), Japan Atomic Energy Agency, Tokai-mura, Japan

${ }^{4}$ Petersburg Nuclear Physics Institute, NRC Kurchatov Institute, 188300 Gatchina, Russia

${ }^{5}$ KU Leuven, Instituut voor Kern- en Stralingsfysica, 3001 Leuven, Belgium

${ }^{6}$ Physics Department, Faculty of Science, Jouf University, Aljouf, Saudi Arabia

${ }^{7}$ The University of Manchester, School of Physics and Astronomy, Oxford Road, M13 9PL Manchester, United Kingdom

${ }^{8}$ Department of Nuclear Physics and Biophysics, Comenius University in Bratislava, 84248 Bratislava, Slovakia

${ }^{9}$ Max-Planck-Institut für Kernphysik, Saupfercheckweg 1, 69117 Heidelberg, Germany

${ }^{10}$ Department of Physics, University of Liverpool, Liverpool, L69 7ZE, United Kingdom

${ }^{11}$ Center for Nuclear Study (CNS), Graduate School of Science, The University of Tokyo, Japan

${ }^{12}$ Institut für Physik, Johannes Gutenberg-Universität Mainz, Mainz, D-55128, Germany

(Received 18 February 2021; accepted 2 August 2021; published 23 August 2021; corrected 20 October 2021)

\begin{abstract}
A study of the laser-ionized and mass-separated neutron-deficient isotopes ${ }^{176,177,179} \mathrm{Au}$ was performed using the Resonance Ionization Laser Ion Source and the Windmill detection setup at ISOLDE, CERN. New and improved data on complex fine-structure $\alpha$ decays of the three isotopes were deduced, providing insight into the low-lying levels in the daughter nuclei ${ }^{172,173,175}$ Ir. New information on the properties of $\beta$-decay daughter products ${ }^{177,179} \mathrm{Pt}$ was also obtained. From the first in-source laser spectroscopy measurements of the hyperfine structure in the atomic $267.6-\mathrm{nm}$ transition of ${ }^{176} \mathrm{Au}$, the nuclear magnetic moments for both high- and low-spin $\alpha$-decaying states were deduced. Together with the values determined from the additivity relations, they were used to propose the most probable spins and configurations for both states. The $\alpha$-decay branching ratios were determined as $b_{\alpha}\left({ }^{176} \mathrm{Au}^{\mathrm{ls}}\right)=58(5) \%$ and $b_{\alpha}\left({ }^{176} \mathrm{Au}^{\mathrm{hs}}\right)=29(5) \%$.
\end{abstract}

DOI: 10.1103/PhysRevC.104.024326

\section{INTRODUCTION}

The neutron-deficient nuclei around the closed proton shell at $Z=82$ and neutron midshell at $N=104$ exhibit some of the best examples of shape coexistence at low energy $[1,2]$. One of the clear manifestations of this phenomenon is provided by the mean-squared nuclear charge radii of the ground states (g.s.) and isomeric states in the lightest gold

\footnotetext{
*Andrei.Andreyev@york.ac.uk

${ }^{\dagger}$ Present address: TRIUMF, Vancouver, BC V6T 2A3, Canada.

${ }^{\ddagger}$ Present address: Belgian Nuclear Research Center SCKCEN, Boeretang 200, B-2400 Mol, Belgium.

§Present address: Department of Physics, University of York, York, YO10 5DD, United Kingdom.
}

Published by the American Physical Society under the terms of the Creative Commons Attribution 4.0 International license. Further distribution of this work must maintain attribution to the author(s) and the published article's title, journal citation, and DOI. isotopes $(Z=79)$ studied by means of the laser spectroscopy experiments. In particular, a sharp increase in the charge radius and quadrupole deformation was deduced for the ground states of ${ }^{183-186} \mathrm{Au}$, in comparison to weakly deformed heavier gold isotopes [3-7]. The laser spectroscopy data complement a large treasury of nuclear spectroscopy data, available from particle and in-beam $\gamma$-decay studies; see, e.g., Refs. [8-19].

The present investigation is a part of an extended experimental campaign performed by our collaboration at the ISOLDE facility [20] at CERN to study the charge radii and decay properties in the gold isotopic chain. These experiments use the unique capabilities to produce intense and purified beams of short-lived isotopes, provided by the combination of resonant laser ionization and mass separation at ISOLDE. This method often allows us to study weak decay branches unattainable in previous experiments. Furthermore, due to the use of versatile detection setups that provide both direct ion counting and measurements of their $\alpha, \beta$, and $\gamma$ decays, more detailed information can be obtained in comparison to earlier studies. The first results from these studies, mostly with an emphasis on the laser spectroscopy of the 
isotopes ${ }^{177-180,182,187,191,193,195} \mathrm{Au}$, have been discussed in Refs. [15,18,19,21,22].

This work reports on the nuclear spectroscopy data for ${ }^{176,177,179} \mathrm{Au}$, including the first laser spectroscopy measurements for ${ }^{176} \mathrm{Au}$, that lies more than 20 neutrons away from the stable nuclide ${ }^{197} \mathrm{Au}$. The structure of the paper is as follows: A brief description of the experimental method is given in Sec. II, followed by the presentation of the data for ${ }^{179,177,176} \mathrm{Au}$ in Secs. III A-III C. Discussions of results for each isotope and their daughter products are provided in respective parts of Sec. IV.

\section{EXPERIMENT}

The data presented in this work originate from the same experiments as described in Ref. [15], and therefore only the most relevant details will be given here. The ${ }^{176,177,179} \mathrm{Au}$ nuclei were produced in spallation reactions induced by a 1.4-GeV proton beam with a maximum current of $2 \mu \mathrm{A}$ from the CERN PS Booster, impinging on a $50 \mathrm{~g} / \mathrm{cm}^{2}$ thick $\mathrm{UC}_{\mathrm{x}}$ target. The spallation products diffused out of the hot target $\left(T \approx 2000-2200^{\circ} \mathrm{C}\right.$ ) and effused as neutral atoms into the hot ion cavity of the Resonance Ionization Laser Ion Source (RILIS) [23].

The gold atoms were ionized within this cavity when the laser beams were frequency tuned to the three-step gold ionization scheme, shown in Fig. 1 of Ref. [15]. For the hyperfine structure (hfs) measurements and isomer separation for ${ }^{176,177} \mathrm{Au}$, described in Secs. III B and IIIC, the first step 267.6-nm $6 s{ }^{2} S_{1 / 2} \rightarrow 6 p{ }^{2} P_{1 / 2}$ atomic transition was scanned by a frequency-tripled titanium sapphire (Ti:Sa) laser in a narrowband mode, with a typical full width half-maximum (FWHM) bandwidth of $\approx 600 \mathrm{MHz}$ before tripling. Two broadband dye lasers (FWHM bandwidth of $\approx 20 \mathrm{GHz}$ ) were used for the second and third excitation steps.

After selective ionization, the ions were accelerated by a $30-\mathrm{kV}$ electrostatic potential and mass separated according to their mass-to-charge ratio using the General Purpose Separator (GPS). The ion beam of the isotope of interest was then transported to the Windmill (WM) decay station for detailed $\alpha$ - and $\beta$-decay studies. The WM detection system used in this study is shown in Fig. 1 of Ref. [24]. The ion beam passed through a central hole of an annular surface-barrier silicon detector ( $\mathrm{Si1}$ ) and was implanted into one of ten thin carbon foils of $20 \mu \mathrm{g} / \mathrm{cm}^{2}$ thickness, mounted on a rotatable wheel. A second surface-barrier silicon detector (Si2) was positioned $\approx 5 \mathrm{~mm}$ behind the implantation foil. The total $\mathrm{Si} 1+\mathrm{Si} 2$ detection efficiency for $\alpha$ particles was $28(3) \%$ at this "implantation" position. Two weak ${ }^{241}$ Am sources were installed on the WM wheel for the initial calibration of the detectors, while the $\alpha$-decay lines of specific isotopes produced in the experiment were used for the final calibration. Typical FWHM energy resolutions of $\approx 25$ and $\approx 30 \mathrm{keV}$ were observed for $\alpha$ decays in the energy region of 5-6 MeV for $\mathrm{Si} 1$ and $\mathrm{Si} 2$, respectively.

A single-crystal low-energy germanium detector (LEGe) with a thin beryllium window was placed outside the WM chamber behind the $\mathrm{Si} 2$ detector, along the axis of the beam. The WM aluminum back wall had a thickness of $1 \mathrm{~mm}$ at this position, allowing $\gamma$ rays with energies down to $\approx 30 \mathrm{keV}$ to be measured. The typical FWHM energy resolution and detection efficiency of the LEGe at $121.8 \mathrm{keV}$ were $1.1 \mathrm{keV}$ and $7.7 \%$, respectively. The detector was calibrated using ${ }^{152} \mathrm{Eu},{ }^{137} \mathrm{Cs},{ }^{133} \mathrm{Ba}$, and ${ }^{60} \mathrm{Co}$ sources.

\section{EXPERIMENTAL RESULTS}

$$
\text { A. }{ }^{179} \mathrm{Au}
$$

\section{Previously known data for $\alpha$ decay of ${ }^{179} \mathrm{Au}$}

The first decay data for ${ }^{179} \mathrm{Au}$ originate from the studies by Siivola [25], where the values of $E_{\alpha}=5848(5) \mathrm{keV}$ and $T_{1 / 2}=7.2(5) \mathrm{s}$ were measured. ${ }^{1}$ The only reported $\alpha$ decay branching ratio, $b_{\alpha}\left({ }^{179} \mathrm{Au}\right)=22.0(9) \%$, was deduced in Ref. [27]. Our recent laser spectroscopy study at ISOLDE has unambiguously established the g.s. spin and parity as $I^{\pi}\left({ }^{179} \mathrm{Au}\right)=1 / 2^{+}[15]$.

The properties of the $\alpha$-decay daughter nucleus ${ }^{175} \mathrm{Ir}$ were recently reviewed in Ref. [28], where an $M 1,26.1(4)-\mathrm{keV}$ $\gamma$-ray decay feeding the newly established $I^{\pi}=\left(1 / 2^{+}\right)$g.s. of ${ }^{175} \mathrm{Ir}$ was observed for the first time. The existence of this decay is crucial for the understanding of the data from the present work.

\section{2. ${ }^{179} \mathrm{Au} \alpha$ decay}

The combined singles $\alpha$-decay spectrum, measured in Si1 and $\mathrm{Si} 2$ at the implantation point for the run at the GPS mass setting of $A=179$, is shown in Fig. 1(a). The spectrum highlights the strength of our experimental method, which provides a uniquely clean sample, whereby only the $\alpha$ decays of the parent ${ }^{179} \mathrm{Au}$ and of its $\beta$-decay daughter ${ }^{179} \mathrm{Pt}$ have been observed. The total number of $\alpha$ decays of ${ }^{179} \mathrm{Au}$ observed in the $\alpha$-energy region of $5500-5900 \mathrm{keV}$ is $\approx 2.8 \times 10^{5}$.

The calibration of the silicon detectors was performed using $\alpha$ decays from ${ }^{202} \mathrm{Fr}(7238(5) \mathrm{keV}$ [30]), its $\alpha$-decay daughter ${ }^{198}$ At $(6749.0(34) \mathrm{keV}$, being a weighted average from ENSDF [31]), and the $\beta$-decay daughter of ${ }^{178} \mathrm{Au}$, ${ }^{178} \mathrm{Pt}$ (5446(3) keV [32]), taken in short measurements before the run at $A=179$. With this calibration, our value of $E_{\alpha}\left({ }^{179} \mathrm{Au}\right)=5848(4) \mathrm{keV}$ matches well to $5848(5) \mathrm{keV}$ from Ref. [25].

The $5848-\mathrm{keV}$ decay is considered as the ${ }^{179} \mathrm{Au} \rightarrow$ ${ }^{175} \mathrm{Ir}$ g.s. $\rightarrow$ g.s. decay, similar to the previous studies of ${ }^{179} \mathrm{Au}$. A low-intensity $(<0.2 \%)$ higher energy tail of the $5848-\mathrm{keV}$ peak is most likely due to the random summing in the silicon detectors of the Windmill with the high background from $\beta$ decays of longer lived activities which were accumulated in the WM chamber in this and previous runs.

Three newly observed $\alpha$ decays at 5718(10), 5705(15), and $5600(10) \mathrm{keV}$ have been attributed to the f.s. decay of

\footnotetext{
${ }^{1} \mathrm{~A}$ weighted average value presently cited by Evaluated Nuclear Structure Data File (ENSDF) is $T_{1 / 2}=7.1(3)$ s [26], while the original $\alpha$-decay energy is still kept there.
} 

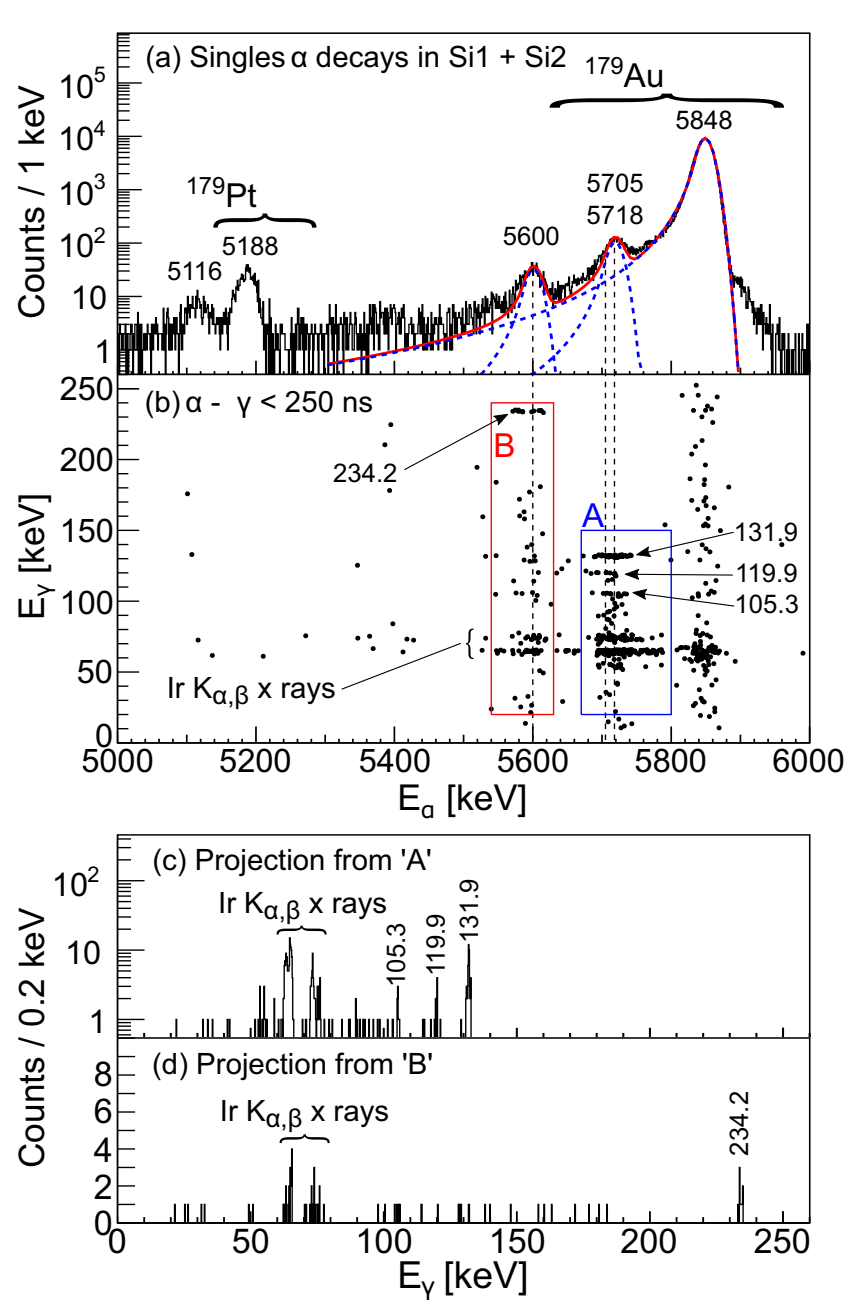

FIG. 1. (a) $\alpha$-decay energy spectrum registered in $\mathrm{Si} 1+\mathrm{Si} 2$ for the runs at mass $A=179$. The peaks are labeled with their energies in $\mathrm{keV}$ and the nuclides they originate from. The fitting of the peaks for ${ }^{179} \mathrm{Au}$ with the Crystal Ball function is shown by the blue dashed line, and the sum of the individual curves is given by the red line. (b) $\alpha-\gamma$ coincidences from panel (a) for $\gamma$-ray energies up to $250 \mathrm{keV}$ with a coincidence timing gate $\Delta T(\alpha-\gamma) \leqslant 250 \mathrm{~ns}$. (c) Projection on the $E_{\gamma}$ axis from within the blue-colored shape "A" in panel (b). Panel (d) is the same as panel (c) but for the events within the rectangle "B" from panel (b).

${ }^{179} \mathrm{Au}$, based on the $\alpha-\gamma$ coincidence analysis. The fitting with Crystal Ball functions [33], which account for the shapes and low-energy tails of the peaks, was used to deduce the intensities of the peaks.

An $\alpha-\gamma$ coincidence matrix for prompt $\gamma$ rays registered in the LEGe following $\alpha$ decays from Fig. 1(a) is shown in Fig. 1(b). A prompt time gate of $\Delta T(\alpha-\gamma) \leqslant 250$ ns was used. Due to a large intensity of the main $5848-\mathrm{keV}$ peak in Fig. 1(a), a small number of $\gamma$ decays and $K_{\alpha, \beta}$ x rays are seen in random coincidences with this $\alpha$ decay.

The projections on the $E_{\gamma}$ axis of events in the regions denoted as "A" and "B" in Fig. 1(b) are shown in Figs. 1(c) and 1(d) respectively. They contain the 105.3(6)-, 119.9(6)-,

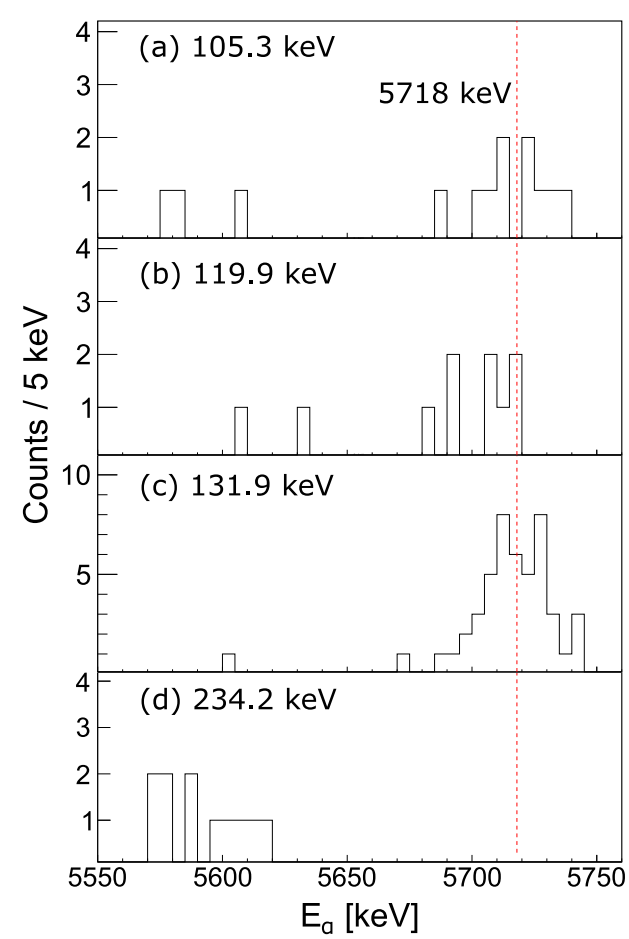

FIG. 2. Projections on the $\alpha$-energy axis from the $\alpha-\gamma$ coincidences plot in Fig. 1(b) using a $\pm 1.5-\mathrm{keV}$ gate on the $\gamma$ ray indicated in the top left of each panel. The vertical red dotted line indicates the centroid of the 5718-keV peak in coincidence with the 105.3- and 131.9-keV $\gamma$ decays.

131.9(4)-, and 234.2(6)-keV $\gamma$ rays, along with iridium $K_{\alpha, \beta}$ $\mathrm{x}$ rays.

Figure 2 shows the $\alpha$-energy projections by gating on the four $\gamma$ rays seen in Fig. 1(b). The 5718-keV decay is in coincidences with the 105.3- and 131.9-keV $\gamma$ rays, as proven by Figs. 2(a) and 2(c), respectively. The $Q_{\alpha \text {,tot }}=$ $Q_{\alpha}(5718)+E_{\gamma}(131.9)=5981(10) \mathrm{keV}$ is in good agreement with $Q_{\alpha}(5848)=5982(4) \mathrm{keV}$, establishing a new excited state at $131.9(4) \mathrm{keV}$ in ${ }^{175} \mathrm{Ir}$; see Fig. 3. The 105.3$\mathrm{keV} \gamma$ ray is assigned as a decay from the $131.9-\mathrm{keV}$ level to the excited state at $26.1(4) \mathrm{keV}$ in ${ }^{175} \mathrm{Ir}$, which was identified in the recent study [28] at the RITU gas-filled separator. Therefore, our data provide an independent, albeit indirect, confirmation for the existence of this state. The strong conversion of the 26.1-keV M1 transition $\left(\alpha_{\text {tot,exp }}=76(14)\right.$ [28]) along with negligible $\gamma$-ray efficiency of the WM setup at such low energies, explains the nonobservation of this decay in our work.

A weak $5705-\mathrm{keV}$ decay is seen in coincidence with the 119.9-keV $\gamma$ ray [Fig. 2(b)]. The $Q_{\alpha, \text { tot }}=Q_{\alpha}(5705)+$ $E_{\gamma}(119.9)=5955(15) \mathrm{keV}$ is in agreement with $Q_{\alpha, \text { tot }}=$ $Q_{\alpha}(5718)+E_{\gamma}(105.3)=5954(10) \mathrm{keV}$, which implies that the $\alpha(5705)-\gamma(119.9)$ and the $\alpha(5718)-\gamma(105.3)$ decay branches feed the same state at $26.1 \mathrm{keV}$. Therefore, we assign the $119.9-\mathrm{keV} \gamma$ ray as proceeding from a new excited state at 146.0(7) keV to the 26.1-keV state in ${ }^{175} \mathrm{Ir}$ (see Fig. 3).

A $5600-\mathrm{keV}$ decay is seen in coincidence with a 234.2$\mathrm{keV} \gamma$ ray [Fig. 2(d)]. The value of $Q_{\alpha, \text { tot }}=Q_{\alpha}(5600)+$ 


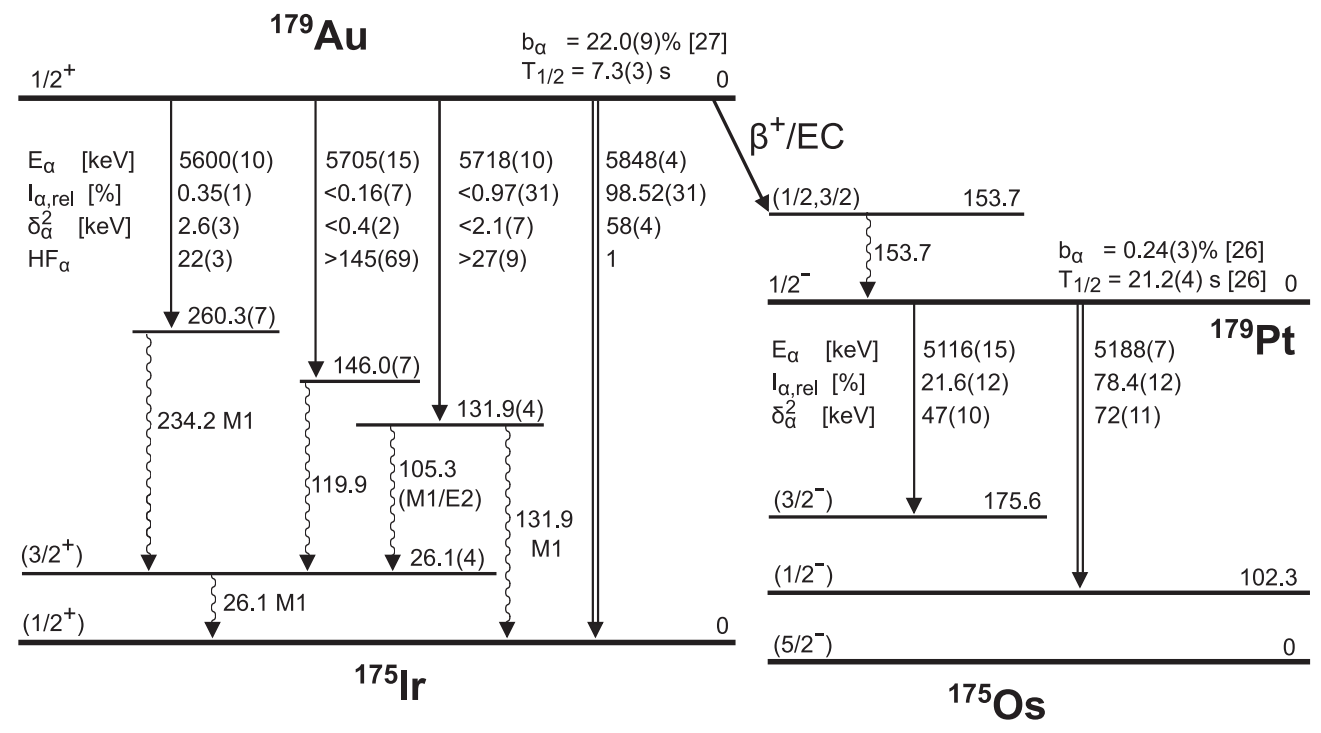

FIG. 3. The decay scheme for ${ }^{179} \mathrm{Au}$ deduced in this study. Shown are the $\alpha$-decay energies $E_{\alpha}$, relative intensities $I_{\alpha, \text { rel }}$, reduced $\alpha$-decay widths $\delta_{\alpha}^{2}$, calculated using the Rasmussen approach [29], and hindrance factors $H F_{\alpha}$ relative to the unhindered g.s. $\rightarrow$ g.s. 5848-keV decay. The 26.1-keV transition was not seen in our study, but its existence was proven in Ref. [28]; see the main text for details. The values taken from the literature are labeled with the respective references in square brackets and were used for calculations of $\delta_{\alpha}^{2}$ values shown in the figure. The $\gamma$ rays de-exciting the 102.3 - and $175.6-\mathrm{keV}$ states in ${ }^{175} \mathrm{Os}$ are known, but not shown in the plot for clarity.

$E_{\gamma}(234.2)=5962(10) \mathrm{keV}$ is within the uncertainty of the $Q_{\alpha, \text { tot }}$ value for the $\alpha(5718)-\gamma(105.3)$ coincidence pair. This implies that the $234.2-\mathrm{keV}$ decay feeds the $26.1-\mathrm{keV}$ excited state, which establishes a new excited state at 260.3(7) keV in ${ }^{175} \operatorname{Ir}$ (Fig. 3).

Previous work by Raddon et al. [34], tentatively suggested the presence of a weak f.s. decay at $5810 \mathrm{keV}$ in ${ }^{179} \mathrm{Au}$ based on its correlations with the $\alpha$ decay of parent nucleus ${ }^{183} \mathrm{Tl}$, but no relevant spectrum was shown in their work. However, no evidence of this decay has been seen in our data, although it might be hidden under the tail from the dominant $5848-\mathrm{keV}$ decay.

\section{Multipolarity of the $\gamma$ rays observed in ${ }^{175} \mathrm{Ir}$}

The prompt nature of the 105.3-, 119.9-, 131.9-, and 234.2$\mathrm{keV} \gamma$ rays in Fig. 1(b) limits their possible multipolarity to $E 1, M 1$, or $E 2$.

By comparing the number of 234.2-keV $\gamma$ rays and $\operatorname{Ir} K_{\alpha, \beta}$ $\mathrm{X}$ rays in Fig. 1(d), after a correction for the $\gamma$-ray efficiency, a $K$-shell internal conversion coefficient of $\alpha_{\mathrm{K}, \exp }(234.2)=$ 0.8 (3) was deduced. This establishes a most probable pure M1 multipolarity for this $\gamma$ ray, as only this multipolarity has a theoretical $K$-shell internal conversion coefficient $\left[\alpha_{\mathrm{K}, \mathrm{th}}(M 1)=0.46\right]$, which is comparable with the experimental value; see Table I. However, a possibility of a mixed $E 2 / M 1$ multipolarity cannot be fully ruled out within the experimental uncertainty. In turn, the above inference leads to a positive parity for the $260.3-\mathrm{keV}$ state, based on the presumed $\left(3 / 2^{+}\right)$assignment for the $26.1-\mathrm{keV}$ level from Ref. [28].

A direct application of this method for the 105.3-, 119.9-, and 131.9-keV $\gamma$ rays in Fig. 1(c) is not possible, as the coincident 5705- and 5718-keV $\alpha$ decays have comparable energies, and thus no suitable gate on a specific $\alpha-\gamma / \mathrm{x}$-ray pairs can be applied. Nevertheless, the determination of the multipolarity for the $131.9-\mathrm{keV}$ decay can be performed, based on the following method. First, we assumed an $M 1 \mathrm{mul}$ tipolarity for both the 105.3- and 119.9-keV transitions, which would provide their highest $K$-shell conversion coefficients, by accounting for their prompt character (see Table I). The latter, in turn, would lead to their highest contribution to the Ir $K_{\alpha, \beta}$ x rays in Fig. 1(c). By subtracting this expected contribution from the total number of $\operatorname{Ir} K_{\alpha, \beta}$ X rays in Fig. 1(c), a lower limit for the number of Ir $K_{\alpha, \beta}$ x rays from solely 131.9-keV decay was calculated, based on which a lower limit of $\alpha_{\mathrm{K}, \exp }(131.9) \geqslant 2.5$ was deduced. The comparison with theoretical values in Table I uniquely establishes an $M 1$ mulipolarity for the 131.9-keV transition and the positive parity for the 131.9-keV excited state.

The above derivation establishes that the majority of the Ir $K_{\alpha, \beta} \times$ rays in Fig. 1(c) originate from the conversion of the 131.9-keV transition. Due to this, and because of the low number of 105.3- and 119.9-keV decays, their unique multipolarity assignment via the above-applied method is not

TABLE I. Experimental, $\alpha_{\mathrm{K}, \exp }$, and theoretical, $\alpha_{\mathrm{K}, \mathrm{th}}, K$-shell internal conversion coefficients for $\gamma$ rays in ${ }^{175} \mathrm{Ir}$. BrIcc calculations [35] were used for the latter.

\begin{tabular}{lcccc}
\hline \hline$E_{\gamma}, \mathrm{keV}$ & $\alpha_{\mathrm{K}, \exp }$ & & $\alpha_{\mathrm{K}, \text { th }}[35]$ & \\
& & $E 1$ & $M 1$ & $E 2$ \\
\hline 105.3 & & 0.28 & 4.35 & 0.7 \\
119.9 & & 0.20 & 3.00 & 0.56 \\
131.9 & $>2.5$ & 0.16 & 2.29 & 0.46 \\
234.2 & $0.8(3)$ & 0.038 & 0.46 & 0.11 \\
\hline \hline
\end{tabular}




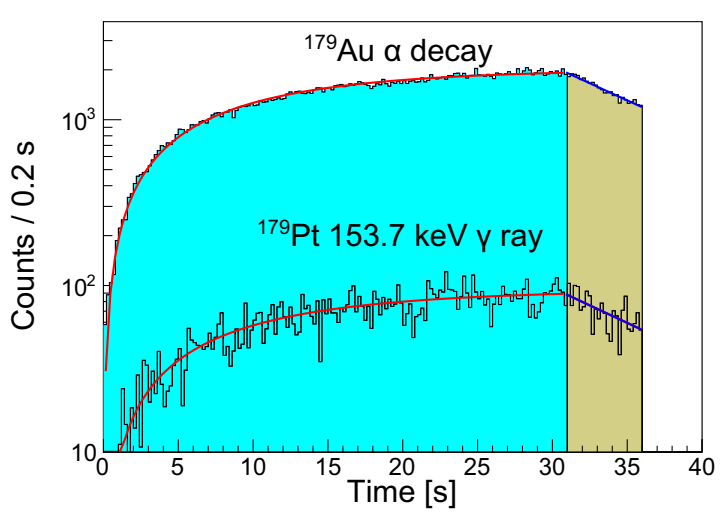

FIG. 4. The time distribution for $\alpha$ decays of ${ }^{179} \mathrm{Au}$ as measured by $\mathrm{Si} 1+\mathrm{Si} 2$ detectors at the implantation position and for the 153.7$\mathrm{keV} \gamma$ decay, following $\beta$ decay of ${ }^{179} \mathrm{Au}$. A sequence of 36-s-long implantation-decay cycles was implemented in which the beam was implanted for $30.89 \mathrm{~s}$ (shaded in light blue) followed by the closure of the ISOLDE beam gate for $5.11 \mathrm{~s}$ (shaded in brown), during which the pure decay of the implanted activity was measured. At the end of each 36-s cycle, the wheel of the WM was rotated and a fresh foil placed into the implantation position for the next measurement cycle. The decay part of each distribution was fitted with an exponential function, from which the values of $T_{1 / 2}\left({ }^{179} \mathrm{Au}\right)=7.3(3) \mathrm{s}$ and 7.1(12) s are calculated for $\alpha$ and $\gamma$ decays, respectively.

possible. However, from the decay scheme in Fig. 3, we can conclude that the $105.3-\mathrm{keV}$ decay has either an $M 1$ or $E 2$ multipolarity (or their mixture), as it does not involve a parity change.

\section{Half-life of ${ }^{179} \mathrm{Au}$ determined from its $\alpha$ decay}

The half-life determination of ${ }^{179} \mathrm{Au}$ has been performed with the grow-in/decay method, presented in Fig. 4. The histogram with larger statistics shows the time distribution of the 5500- to 5900-keV $\alpha$ decays of ${ }^{179} \mathrm{Au}$ from Fig. 1(a). By fitting the decay part of the time distribution with an exponential function, a half-life of 7.3(3) s was deduced, which is in good agreement with the evaluated value of 7.1(3) s from ENSDF [26].

\section{5. ${ }^{179} \mathrm{Au} \rightarrow{ }^{179} \mathrm{Pt} \beta$-decay branch}

The 5188(7)-keV $\alpha$ decay of ${ }^{179} \mathrm{Pt}$ in Fig. 1(a) is consistent, within the uncertainty, with the literature value of 5195(9) $\mathrm{keV}$, being a weighted average from ENSDF [26]. This decay is known to feed an $I^{\pi}=\left(1 / 2^{-}\right)$excited state at $102.3 \mathrm{keV}$ in ${ }^{175}$ Os [36].

Based on the absence of any $\alpha$-decaying contaminants in Fig. 1(a), and on the $Q_{\alpha}$-value analysis, we assigned the 5116(15)-keV peak as a new f.s. decay of ${ }^{179} \mathrm{Pt}$ to the known $\left(3 / 2^{-}\right)$excited state at $175.6 \mathrm{keV}$ in ${ }^{175} \mathrm{Os}$ (see Fig. 3). The relative intensities of the 5188- and 5116-keV decays were deduced as 78.4(12)\% and 21.6(12)\%, respectively. While the $\gamma$-ray decays from both the $102.3-$ and $175.6-\mathrm{keV}$ states are known from previous studies, they were not observed in our data [see Fig. 1(b)], both due to low statistics and expected relatively high internal conversion coefficients for these transitions.

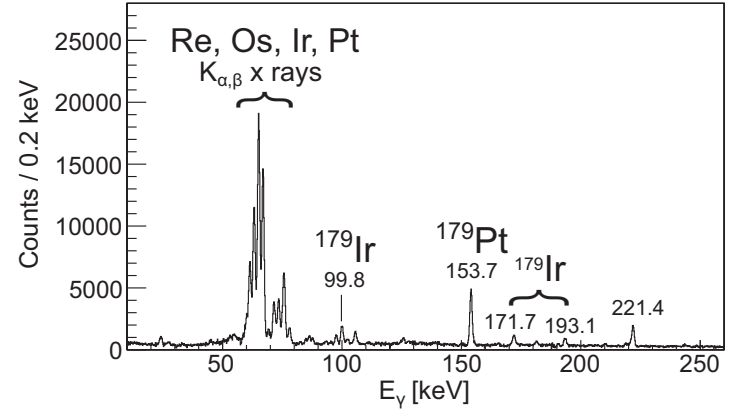

FIG. 5. Background-subtracted $\gamma$-ray singles spectrum in LEGe for the runs at $A=179$, with transitions related to the daughter products of ${ }^{179} \mathrm{Au}$ marked with their energies in keV. Several known $\gamma$ rays in ${ }^{179} \mathrm{Ir}$ are also labeled. The $221-\mathrm{keV}$ transition has a longhalf-life, and its origin could not be established in the present study.

Figure 5 shows the background-subtracted $\gamma$-ray spectrum from the LEGe measured at the $A=179$ ISOLDE mass setting. A background spectrum was taken shortly before the ${ }^{179} \mathrm{Au}$ run. Several known $\gamma$-ray transitions from ${ }^{179} \mathrm{Ir}$, which is the $\beta$-decay granddaughter product of ${ }^{179} \mathrm{Au}$, were clearly identified in the spectrum. A prominent $\gamma$ ray at $153.7 \mathrm{keV}$ has a half-life of 7.1(12) s, derived as shown in Fig. 4. Within the experimental uncertainty, this value matches to 7.3(3) $\mathrm{s}$ deduced for the $\alpha$ decay of ${ }^{179} \mathrm{Au}$. This implies that the 153.7$\mathrm{keV}$ decay originates from an excited state in ${ }^{179} \mathrm{Pt}$, populated by the $\beta$ decay of ${ }^{179} \mathrm{Au}$. This suggestion is also based on the purity of the implanted sample and on the fact that none of the $A=179$ daughter products have a comparable half-life. No $\gamma$ rays were found in coincidence with the $153.7-\mathrm{keV}$ transition, and thus we tentatively placed it as feeding directly the $1 / 2^{-}$ ground state of ${ }^{179} \mathrm{Pt}$ (see Fig. 3). Based on $\beta$-decay selection rules, the most probable spin of the $153.7-\mathrm{keV}$ state is $(1 / 2)$ or $(3 / 2)$.

This $153.7-\mathrm{keV}$ decay should be different from the known $153.8-\mathrm{keV} 7 / 2^{-} \rightarrow 5 / 2^{-}$transition from the $241.2-\mathrm{keV}$ excited state in ${ }^{179} \mathrm{Pt}$, as no subsequent $87.4-\mathrm{keV} \gamma$ ray is seen in our data, which would be expected based on the decay scheme in ENSDF [26]. Furthermore, the $\beta$ decay from the $1 / 2^{+}$ ground state in ${ }^{179} \mathrm{Au}$ to the $241.2-\mathrm{keV} 7 / 2^{-}$state in ${ }^{179} \mathrm{Pt}$ is highly unlikely. On the same grounds, the $153.7-\mathrm{keV}$ decay should be different from the known $153.7-\mathrm{keV}$ transition from the $298.5-\mathrm{keV}\left(9 / 2^{+}\right)$state to the $144.8-\mathrm{keV}\left(7 / 2^{-}\right)$excited state in ${ }^{179} \mathrm{Pt}[26]$.

\section{B. ${ }^{177} \mathrm{Au}$}

\section{Previously known data}

The first identification of the isotope ${ }^{177} \mathrm{Au}$ was performed in Ref. [37]. Since then, it has been studied by a number of particle decay and in-beam spectroscopy experiments; see the recent data evaluation [38]. Two $\alpha$-decaying states are known in this nucleus: an $I^{\pi}=1 / 2^{+}$g.s. with $T_{1 / 2}=1.501(20) \mathrm{s}$ and $b_{\alpha}=40(6) \%$ and an excited $I^{\pi}=11 / 2^{-}$isomeric state with $T_{1 / 2}=1.193(13) \mathrm{s}$ and $b_{\alpha}=66(10) \%$. The evaluated halflife values are from Ref. [38], the branching ratios are from Ref. [10], and the spin assignments are from Refs. [15,18]. 


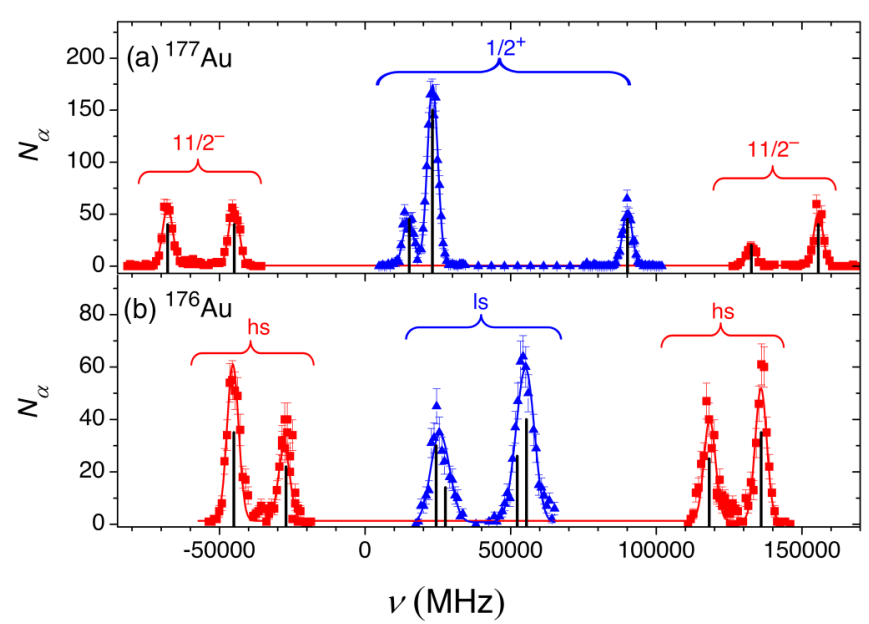

FIG. 6. Typical examples of the hfs spectra (filled symbols) measured by $\alpha$ decay counting with WM, by using the 267.6-nm transition for (a) ${ }^{177} \mathrm{Au}^{\mathrm{g}, \mathrm{m}}$ (data are from Refs. [15,18]) and (b)

${ }^{176} \mathrm{Au}$ from the present study. The zero frequency corresponds to the centroid position of the hfs for the reference isotope ${ }^{197} \mathrm{Au}$. For ${ }^{176} \mathrm{Au}$, the solid lines depict Voigt-profile fits to the data with the assumptions of $I=9$ for the high-spin state in ${ }^{176} \mathrm{Au}$ and $I=3$ for the low spin; the respective theoretical positions of the hfs components are shown by the vertical black lines. Fits with different assignments ( $I=7,8$ for high spin and $I=2,4,5$ for low spin) are indistinguishable from those shown in the figure. The four theoretically expected hfs components for low-spin ${ }^{176} \mathrm{Au}$ were not fully resolved, and therefore only two broader peaks were observed, each of them involving two overlapping hfs components.

A summary of multiple and consistent $\alpha$-decay energy determinations for both states is also provided in Ref. [38]. The excitation energy of $E^{*}\left(11 / 2^{-},{ }^{177} \mathrm{Au}^{\mathrm{m}}\right)=181.9(4) \mathrm{keV}$ has been recently deduced in Ref. [16], being in agreement with but more precise than a value of $189(15) \mathrm{keV}$ from its first determination in Ref. [10].

Hfs spectra of ${ }^{177} \mathrm{Au}^{\mathrm{g}, \mathrm{m}}$ have been recently measured by our collaboration in Refs. [15,18]; the nuclear spectroscopic data in the present work originate from the same experimental campaign. For the completeness of the present discussion, an example of a full hfs scan for both states, constructed from the hfs spectra shown in Refs. [15,18], is given in Fig. 6(a).

\section{2. $\alpha$-decay data for ${ }^{177} \mathrm{Au}^{\mathrm{g}, \mathrm{m}}$ from the present work}

As seen in Fig. 6(a), the frequency ranges for the ionization of each state are well separated, and therefore their decays could be studied individually, by tuning the lasers to production of either the ground or isomeric state. This isomer-selection technique provides uniquely clean samples, unachievable in the previous experiments.

Figures 7(a) and 7(b) show the combined singles $\alpha$-decay spectra measured in $\mathrm{Si} 1$ and $\mathrm{Si} 2$, with the lasers scanned over the frequencies corresponding to the ground and isomeric states, respectively. The silicon detectors were calibrated using $\alpha$ decays from ${ }^{179} \mathrm{Au}$ and from the $\beta$-decay daughter of ${ }^{178} \mathrm{Au},{ }^{178} \mathrm{Pt}$, taken in measurements shortly before and after the run at $A=177$. The spectra clearly show the main $\alpha$ -

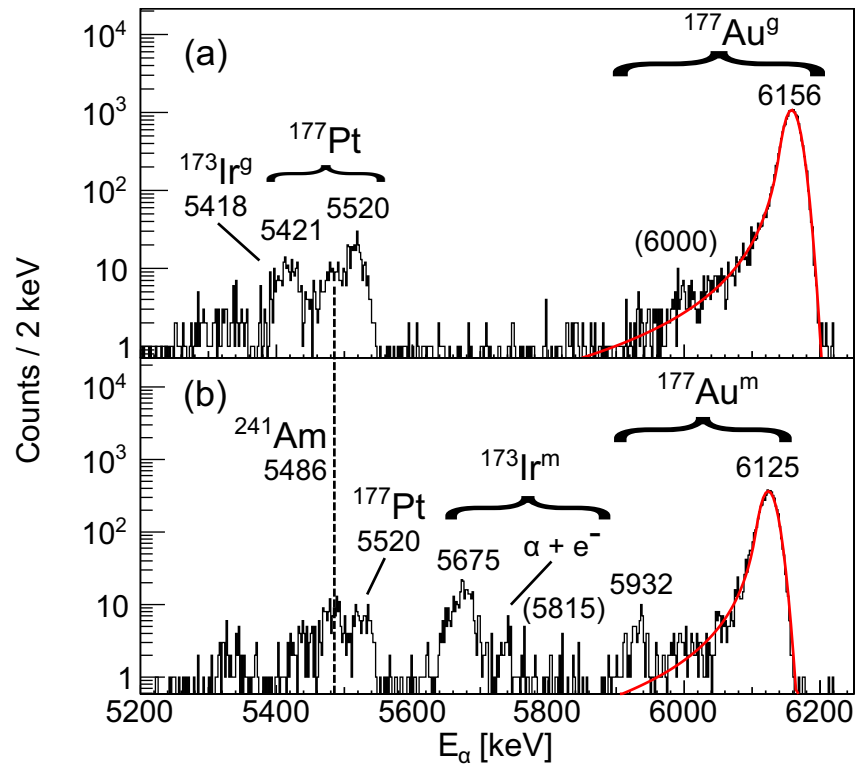

FIG. 7. (a) Combined singles $\alpha$-decay spectrum from $\mathrm{Si} 1+\mathrm{Si} 2$ with the lasers tuned to the ${ }^{177} \mathrm{Au}^{\mathrm{g}}$ hfs frequencies. (b) The same, but with the lasers tuned to the ${ }^{177} \mathrm{Au}^{\mathrm{m}}$ frequency range. The peaks are labeled with their energies in $\mathrm{keV}$ and the nuclides they originate from. The tentative peaks are labeled with their energies in brackets. A small peak at $5486 \mathrm{keV}$ is due to the weak calibration ${ }^{241} \mathrm{Am}$ sources installed on both sides of the WM wheel. The origin of a weak $\approx 5330-\mathrm{keV}$ decay observed in both panels of the figure could not be established. The red lines in both panels are the Crystal Ball fits, similar to those of ${ }^{179} \mathrm{Au}$.

decay peaks from ${ }^{177} \mathrm{Au}^{\mathrm{g}, \mathrm{m}}$ along with those from the $\beta$-decay daughter ${ }^{177} \mathrm{Pt}$. The total numbers of $\alpha$ decays observed in the $\alpha$-energy region of $5900-6200 \mathrm{keV}$ are $1.7 \times 10^{4}$ for of ${ }^{177} \mathrm{Au}^{\mathrm{g}}$ and $5.8 \times 10^{3}$ for ${ }^{177} \mathrm{Au}^{\mathrm{m}}$.

The value of $E_{\alpha}\left({ }^{177} \mathrm{Au}^{\mathrm{g}}\right)=6156(5) \mathrm{keV}$ in Fig. 7(a) is in good agreement with the literature values, e.g., with 6156(6) $\mathrm{keV}$ from Ref. [8]. The $\alpha$ decays from ${ }^{177} \mathrm{Pt}$ at $5520(10) \mathrm{keV}$ (5517(4) $\mathrm{keV}$ [32]) and 5421(10) keV (5423(10) keV [32]) are also in good agreement with previous studies. The 5418(4)$\mathrm{keV} \alpha$ decay of the daughter isotope ${ }^{173} \mathrm{Ir}^{\mathrm{g}}$ [39] is expected to contribute to the $5421-\mathrm{keV}{ }^{177} \mathrm{Pt} \alpha$ decay peak, despite the fact that ${ }^{173} \mathrm{Ir}^{\mathrm{g}}$ has a small $\alpha$-decay branch (4(2)\% [9]) and its intensity is further reduced due to its long half-life of 9.0(8) s and the movement of the wheel of the windmill at the end of each implantation-decay cycle of $\approx 36 \mathrm{~s}$.

Tentative evidence for a weak $6000(20)-\mathrm{keV}$ decay is seen in Fig. 7(a), as a small increase of counts above the smooth Crystal Ball fit, shown by the red line. The $Q_{\alpha}$-value analysis suggests that this decay could feed the known excited state at $155.4(10) \mathrm{keV}$ in ${ }^{173} \mathrm{Ir}$ [40]. An upper limit for its intensity was estimated as $0.23 \%$ relative to the $6156-\mathrm{keV}$ peak. Due to its highly tentative nature, we prefer not show it in the decay scheme in Fig. 8.

In Fig. 7(b), the energy of $E_{\alpha}\left({ }^{177} \mathrm{Au}^{\mathrm{m}}\right)=6125(5) \mathrm{keV}$ is in good agreement with the previous measurements for this $11 / 2^{-} \rightarrow\left(11 / 2^{-}\right)$decay; e.g., a value of $6122(6)-\mathrm{keV}$ was reported in Ref. [8]. By combining the new value of 
(a) ${ }^{177} \mathrm{Au}^{\mathrm{g}}$

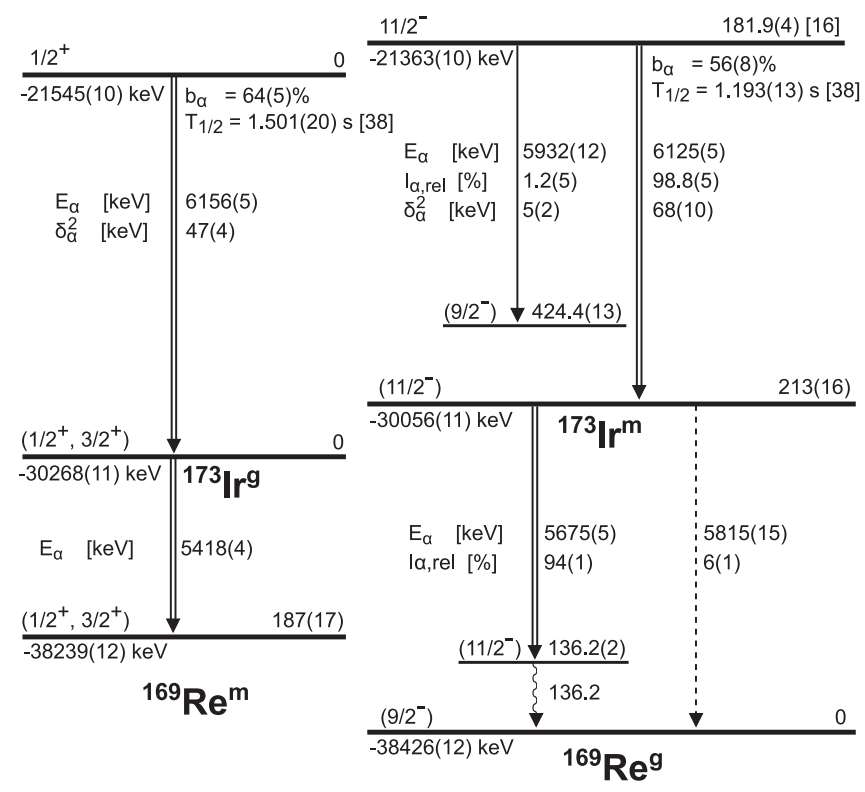

FIG. 8. The decay schemes for ${ }^{177} \mathrm{Au}^{\mathrm{m}, \mathrm{g}} \rightarrow{ }^{173} \mathrm{Ir}^{\mathrm{m}, \mathrm{g}} \rightarrow{ }^{169} \mathrm{Re}^{\mathrm{m}, \mathrm{g}}$. Shown are the $\alpha$-decay energies $E_{\alpha}$, reduced $\alpha$-decay widths $\delta_{\alpha}^{2}$, calculated using the Rasmussen approach [29], relative intensities $I_{\alpha, \text { rel }}$, half-life values $T_{1 / 2}$, and $\alpha$-decay branching ratios $b_{\alpha}$. The values taken from literature are labeled with the respective references in square brackets; they were used for calculating reduced widths. See the main text for the discussion of masses and excitation energies of some states shown in the plot. The de-excitation path of the known 424.4-keV state in ${ }^{173} \mathrm{Ir}$ is not shown for clarity.

$E^{*}\left({ }^{177} \mathrm{Au}^{\mathrm{m}}\right)=181.9(4) \mathrm{keV}$ from Ref. [16] with a reference mass for ${ }^{177} \mathrm{Au}^{\mathrm{g}}$ from the AME2016 [41] and $\alpha$-decay energies measured in the present study, an excitation energy of $E^{*}\left(11 / 2^{-},{ }^{173} \mathrm{Ir}^{\mathrm{m}}\right)=213(16) \mathrm{keV}$ is derived, which is in agreement with 226(18) keV from Ref. [10]. By further propagating the mass analysis to ${ }^{169} \mathrm{Re}^{\mathrm{g}, \mathrm{m}}$, an excitation energy of 187(17) keV is established for ${ }^{169} \mathrm{Re}^{\mathrm{m}}$. The decay scheme of ${ }^{177} \mathrm{Au}$ which includes both the evaluated data and the results from the present experiment is shown in Fig. 8 and will be discussed further in the text.

A weak $\alpha$ decay at 5932(12) keV is also seen in Fig. 7(b). The $Q_{\alpha}$-value analysis establishes that this decay feeds an excited state at $411(16) \mathrm{keV}$ in ${ }^{173} \mathrm{Ir}$, which matches the excitation energy of the known $\left(9 / 2^{-}\right)$state at $424.4(13) \mathrm{keV}$ in ${ }^{173}$ Ir [40]. On these grounds, the $5932-\mathrm{keV}$ decay is assigned as a f.s. decay to the 424.4-keV state (see Fig. 8). No $\gamma$ ray was seen in coincidence with the $5932-\mathrm{keV} \alpha$ decay in this work, most likely due to low statistics.

In Fig. 7(b), the 5675(5)-keV decay is attributed to ${ }^{173} \mathrm{Ir}^{\mathrm{m}}$, as it matches well to the weighted average of $5672(3) \mathrm{keV}$ from previous studies [39]. This decay was seen in prompt coincidences with a single $\gamma$ ray at $136.2 \mathrm{keV}$ (not shown in the plots provided here), confirming the results from Refs. [9,42]. The $K$-shell internal conversion of the $136.2-\mathrm{keV}$ transition leads to the dominant appearance of $\approx 60-\mathrm{keV}$ conversion electrons (CEs), whose energy will sum with the energy of the coincident $5675-\mathrm{keV}$ decay, if both $\alpha$ particle and CE are registered in the same silicon detector of the WM. This effect creates an artificial summing peak at $\approx 5740 \mathrm{keV}$, marked as $\alpha+\mathrm{e}^{-}$in Fig. 7(b).

The energy of a weak group at 5815(15) $\mathrm{keV}$ in Fig. 7(b) matches that of the possible full-energy cross-over $\left(11 / 2^{-}\right) \rightarrow\left(9 / 2^{-}\right)$decay of ${ }^{173} \mathrm{Ir}^{\mathrm{m}}$. On these grounds, it was tentatively placed in the decay scheme as shown in Fig. 8. The relative intensities of the 5675- and 5815-keV decays are $94(1) \%$ and 6(1)\%, respectively.

By using the same method as outlined for ${ }^{179} \mathrm{Au}$, the decay part of the implantation and decay sequence (between 30.89 and $36.0 \mathrm{~s}$ ) for the respective $\alpha$ decays of ${ }^{177} \mathrm{Au}^{\mathrm{g}, \mathrm{m}}$ were fitted using an exponential function. From these fits, the values of $T_{1 / 2}\left({ }^{177} \mathrm{Au}^{\mathrm{g}}\right)=1.37(7) \mathrm{s}$ and $T_{1 / 2}\left({ }^{177} \mathrm{Au}^{\mathrm{m}}\right)=1.27(11) \mathrm{s}$ were extracted. These data are less precise than the literature values, but are in agreement within their respective uncertainties.

The $\alpha$-decay branching ratios of $b_{\alpha}\left({ }^{177} \mathrm{Au}^{\mathrm{m}}\right)=56(8) \%$ and $b_{\alpha}\left({ }^{177} \mathrm{Au}^{\mathrm{g}}\right)=64(5) \%$ were deduced with the method described in the Supplemental Material [43], following the beam implantation pattern modelled as in Ref. [44]. They are shown in Fig. 8 and used in this work for evaluation of respective reduced $\alpha$-decay widths.

$$
\text { C. }{ }^{176} \mathrm{Au}
$$

\section{Previously known data}

For the consistency of the followup discussion, this section briefly summarizes the previously known decay data for ${ }^{176} \mathrm{Au}$. The first identification of the isotope ${ }^{176} \mathrm{Au}$ was reported in the $\alpha$-decay investigation in Ref. [45]. Two $\alpha$-decay lines at 6.26(1) and 6.29(1) MeV and a half-life of 1.25(30) s for the lower energy and higher intensity decay were deduced.

To our knowledge, a $\beta$-decay study of ${ }^{176} \mathrm{Au}$ in Ref. [46] was the first to suggest the existence of two isomeric states in this nucleus. Based on the observed feeding pattern of the states in the daughter ${ }^{176} \mathrm{Pt}$ the authors stated that "it appears that ${ }^{176} \mathrm{Au}$ may also have more than one $\beta$-decaying state, and that they have spins of $\approx 2 \hbar$ and $\approx 8 \hbar$ " (see the discussion of Fig. 7 in Ref. [46]).

The in-beam and $\alpha$-decay work [9] identified several finestructure (f.s.) $\alpha$ decays, originating from two different states in ${ }^{176} \mathrm{Au}$, with the half-lives of $1.36(2)$ and $1.05(1) \mathrm{s}$. The authors proposed spins and parities of $\left(9^{+}\right)$and $\left(3^{-}\right)$for the high-spin (hs) and low-spin (ls) states, respectively, partially based on the $\beta$-decay work [46].

So far, the most detailed f.s. $\alpha$-decay investigation of ${ }^{176} \mathrm{Au}$ was performed at the velocity filter SHIP (GSI), which also summarized all available data; see Tables I and II and Fig. 3 in Ref. [12] and references therein. Based mostly on $\alpha$-decay hindrance factors and systematics of odd-odd isotopes in this region, tentative spin assignments of $\left(8^{+}, 9^{+}\right)$and $\left(3^{-}, 4^{-}\right)$ were proposed for the hs and ls states, respectively.

A dedicated study of the $\alpha$-decay chain ${ }^{180} \mathrm{Tl} \rightarrow$ ${ }^{176} \mathrm{Au}^{\text {ls }} \rightarrow{ }^{172}$ Ir by our collaboration at ISOLDE [47] suggested an $\left(4^{-}, 5^{-}\right)$assignment for ${ }^{176} \mathrm{Au}^{\text {ls }}$, with a reference to the preliminary analysis in the present study. The hs isomer in ${ }^{176} \mathrm{Au}$ could not be populated in Ref. [47].

It is important to mention that, with an exception of work [47], all previous studies of ${ }^{176} \mathrm{Au}$ were performed with the 
TABLE II. The values of $a(6 s)$, the ratios $a(6 p) / a(6 s),{ }^{\text {ref }} \Delta^{A}$, and the magnetic moments deduced in this study for ${ }^{176} \mathrm{Au}{ }^{\mathrm{ls}, \mathrm{hs}}$ for different spin assumptions, $I$, within the approach from Ref. [53] with revised uncertainties according to Ref. [18], and with the RHFA correction following the prescription from Ref. [18].

\begin{tabular}{|c|c|c|c|c|c|c|}
\hline Isotope & Spin $I$ & $a(6 s)[\mathrm{MHz}]$ & $a(6 p) / a(6 s)$ & ${ }^{197} \Delta^{176 \mathrm{hs}}(\%)$ & $\mu_{\exp }\left(\mu_{N}\right),[53]$ & $\mu_{\exp }\left(\mu_{N}\right),[18]$ \\
\hline \multirow{3}{*}{${ }^{176} \mathrm{Au}^{\mathrm{hs}}$} & 10 & $15550(150)$ & $0.1093(30)$ & $4.9(39)$ & $5.36(26)$ & $5.20(20)$ \\
\hline & 9 & $17180(140)$ & $0.1096(30)$ & $5.3(39)$ & $5.33(26)$ & $5.18(20)$ \\
\hline & 7 & $21770(160)$ & $0.1093(30)$ & $4.9(39)$ & $5.25(25)$ & $5.09(19)$ \\
\hline \multirow[t]{3}{*}{${ }^{176} \mathrm{Au}^{\mathrm{lsa}}$} & 5 & $-5060(120)$ & & & $-0.873(55)$ & \\
\hline & 4 & $-6180(140)$ & & & $-0.853(54)$ & \\
\hline & 2 & $-11130(180)$ & & & $-0.767(43)$ & \\
\hline
\end{tabular}

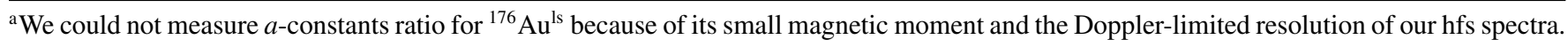
Due to this, we could not determine RHFA; therefore, the $\mu$ values for ${ }^{176} \mathrm{Au}^{\text {ls }}$ were calculated according to the prescription of Ref. [53] only.

use of fusion-evaporation reactions. The presence of a multitude of strongly competing evaporation channels, combined with similar half-lives and $\alpha$-decay energies for ${ }^{176} \mathrm{Au}^{\mathrm{ls} \text {,hs }}$, hampered the data analysis and required a careful application of the $\alpha$-decay correlation technique; see, e.g., Refs. [9,12,48].

Since the relative position of the two states was not yet established, in the following text they will be referred to as "low spin" $\left({ }^{176} \mathrm{Au}^{\mathrm{ls}}\right)$ and "high spin" $\left({ }^{176} \mathrm{Au}^{\text {hs }}\right)$ states. We refer the reader to the evaluation in Ref. [49] for information on the $\alpha$-decaying daughter product ${ }^{172} \mathrm{Ir}$, which will be partially used further in the text.

\section{2. $\alpha$-decay data from the present work}

An example of an hfs spectrum for ${ }^{176} \mathrm{Au}$, measured in the present work for the first time, is shown in Fig. 6(b). Similar to ${ }^{177} \mathrm{Au}^{\mathrm{g}, \mathrm{m}}$, a complete separation of the hfs components for the two states using RILIS was achieved. This allowed us to obtain isomerically pure beams of both isomers, to be further studied via their $\alpha$ and $\gamma$ decays with the WM setup.

For completeness, we mention that an isobaric contamination due to ${ }^{176} \mathrm{Yb}$ was present during these measurements. This rare-earth-metal isotope close to stability is strongly produced in the target and is surface ionized in the hot cavity of the RILIS. We observed numerous $\gamma$-ray lines originating from excited states of this stable isotope, e.g., from its 11.4-s isomeric state, which made the search for $\gamma$ decays following the $\beta$ decay of a more weakly produced ${ }^{176} \mathrm{Au}$ impossible. However, no contribution to $\alpha$-decay data, discussed below, was noted.

Figures 9(a) and 9(b) show $\alpha$-decay energy spectra measured at the ISOLDE mass setting of $A=176$, with the lasers tuned to the ionization of ${ }^{176} \mathrm{Au}^{\text {hs }}$ and of ${ }^{176} \mathrm{Au}^{\text {ls }}$, respectively. The numbers of $\alpha$ decays in the region of $6000-6300 \mathrm{keV}$ are $4.5 \times 10^{3}$ for ${ }^{176} \mathrm{Au}^{\text {hs }}$ and $3 \times 10^{3}$ for ${ }^{176} \mathrm{Au}^{\text {ls }}$. Since the hfs measurements were the main goal of this experiment, the total statistics for ${ }^{176} \mathrm{Au}$ was lower than in the SHIP study [12]. Nevertheless, due to the purity of the $\alpha$-decay spectra, new and improved information was obtained. In particular, while the previously known data for for ${ }^{176} \mathrm{Au}^{\text {hs }}$ were mostly confirmed, an important difference between the present study and the earlier results from Refs. [9,12] was observed for ${ }^{176} \mathrm{Au}^{\mathrm{ls}}$; see the follow-up discussion.
${ }^{176} \mathrm{Au}^{\mathrm{hs}}$ decay. The decay scheme of ${ }^{176} \mathrm{Au}^{\mathrm{hs}}$ compiled from this study and from Refs. [9,12,42] is shown in Fig. 10(a). Our data are in good agreement with those from Refs. [9,12]. Namely, the 6080(5) keV [33(2)\%], 6114(5) keV [60(2)\%], and 6280(10) $\mathrm{keV}$ [7(1)\%] $\alpha$ decays in Fig. 9(a) match well the most recent data from the SHIP study [12], where the respective energies and relative intensities were reported as 6082(7) $\mathrm{keV}$ [26(5)\%], 6117(7) keV [66(5)\%], and $6287(7) \mathrm{keV}[8(1) \%] .^{2}$ The prompt $\alpha(6080)-\gamma(211.6)$ and $\alpha(6114)-\gamma(175.2)$ coincidences were also observed (not shown in this work), confirming the data from Refs. [9,12,42].

The 6212(15)-keV group is proven in Refs. $[9,12]$ as resulting from the $\alpha(6080 / 6114)+$ CEs summing, the coincident conversion electrons arising from the $175.2-$ and $211.6-\mathrm{keV}$ M1 transitions. The summing effect is much smaller in the present study, due to the implantation in an external carbon foil, rather than directly in the silicon detector, as in Refs. [9,12]. The intensity of the 6212-keV line was reassigned to the 6080- and 6114-keV decays, similar to that done in Ref. [12].

The peaks due to the decay products of ${ }^{176} \mathrm{Au}^{\text {hs }}$ are also seen at 5824(10) $\mathrm{keV}$ (5828(3) keV [50]) for ${ }^{172} \mathrm{Ir}^{\text {hs }}$ and 5753(5) keV (5753(3) keV [32]) for ${ }^{176} \mathrm{Pt}$.

We assign the weak 5983(15)-keV peak as a new $\alpha$ decay of ${ }^{172} \mathrm{Ir}^{\mathrm{hs}}$, which corresponds to the full energy crossover decay, parallel to the known $\alpha(5824)-\gamma(162.1, M 1 / E 2)$ decay path; see Ref. [42]. These coincidences were also observed in our data. The $K$-shell internal conversion of the 162.1$\mathrm{keV}$ transition leads to the emission of $\approx 86-\mathrm{keV}$ conversion electrons. The summing of the energy of the 5824-keV decay and of those electrons in the silicon detectors should lead to an artificial peak at $\approx 5910 \mathrm{keV}$, and thus we attribute the 5919(20)-keV peak in Fig. 9(a) to this summing effect. Its intensity is added to that of the $5824-\mathrm{keV}$ decay, leading to the relative intensities of the 5824- and 5983-keV transitions of ${ }^{172} \mathrm{Ir}^{\mathrm{hs}}$ as $93(2) \%$ and 7(2)\%, respectively; see Fig. 10(a).

\footnotetext{
${ }^{2}$ The respective values from the study [9] are 6082(5), 6117(5), and $6287 \mathrm{keV}$; the latter decay was identified tentatively with an upper limit on the relative intensity of $<4 \%$.
} 


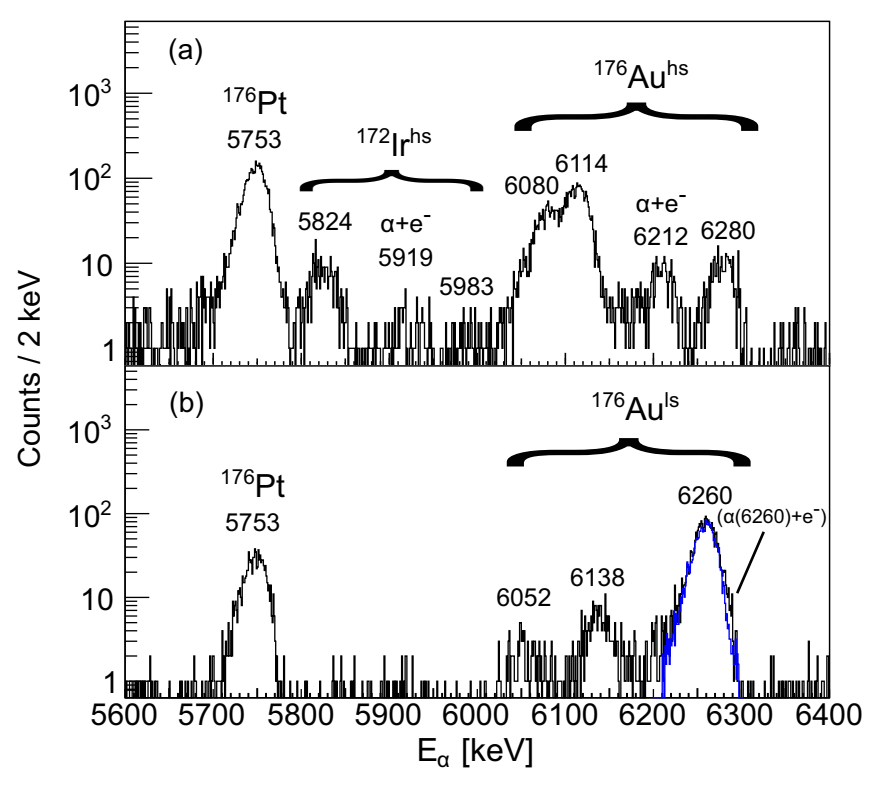

FIG. 9. (a) Combined singles $\alpha$-decay spectrum at $A=176$, from $\mathrm{Si} 1+\mathrm{Si} 2$ with the lasers tuned to the ${ }^{176} \mathrm{Au}^{\mathrm{hs}} \mathrm{hfs}$ frequencies. (b) The same, but with the lasers tuned to the ${ }^{176} \mathrm{Au}^{\text {ls }}$ frequency range. The peaks are labeled with their energies in $\mathrm{keV}$ and the nuclide they originate from. The shifted and normalized $5753-\mathrm{keV}$ peak of ${ }^{176} \mathrm{Pt}$ is shown by the blue histogram in panel (b); see Sec. IV C 3 for discussion of this procedure

${ }^{176} \mathrm{Au}^{\mathrm{ls}}$ decay. The decay scheme of ${ }^{176} \mathrm{Au}^{\mathrm{ls}}$ deduced in the present investigation is shown in Fig. 10(b), with some data taken from the previous studies [9,12,42,47], as will be explained below. The SHIP work identified five $\alpha$-decay branches for ${ }^{176} \mathrm{Au}^{\text {ls }}$ [12], with the dominant $6287(7)-\mathrm{keV}$ $\left(I_{\alpha, \text { rel }}>92 \%\right)$ decay, while only a single $6282-\mathrm{keV}$ decay was reported for ${ }^{176} \mathrm{Au}^{1 \mathrm{~s}}$ in Ref. [9]. However, instead of the 6287-keV (or 6282-keV) decay, a strong 6260(5)-keV decay is observed in the present work for ${ }^{176} \mathrm{Au}^{\text {ls }}$; see Fig. 9(b). We recall that in the SHIP investigation [12] the presence of the $6287-\mathrm{keV}$ peak was deduced via a complex subtraction of a correlated $\alpha$-decay spectrum of ${ }^{176} \mathrm{Au}^{\text {hs }}$ from the total $\alpha$-decay spectrum. In the present study, the $6260-\mathrm{keV}$ peak is clearly seen without any additional analysis. Further discussion of this decay will be given in Sec. IV C 3.

In the SHIP work [12], based on the prompt $\alpha-\gamma$ coincidences analysis, four weak f.s. $\alpha$ decays at 5798(20)-500.0(6) $\mathrm{keV}\left(I_{\alpha, \text { rel }} \leqslant 0.4 \%\right), 6054(20)-236.6(3) \mathrm{keV}\left(I_{\alpha, \text { rel }} \leqslant 1 \%\right)$, $6138(15)-151.5(3) \mathrm{keV}\left(I_{\alpha, \text { rel }} \leqslant 5 \%\right)$, and 6157(20)-126.3(3) $\operatorname{keV}\left(I_{\alpha, \text { rel }} \leqslant 2 \%\right)$ were identified for ${ }^{176} \mathrm{Au}^{\mathrm{ls}}$. Due to high statistics collected for the ${ }^{180} \mathrm{Tl} \rightarrow{ }^{176} \mathrm{Au}^{\text {ls }}$ decay chain, the ISOLDE study [47] provided improved energy data for some of these decays, via the observation of 5798(10)-500.0(3) $\mathrm{keV}, 6054(7)-236.0(2) \mathrm{keV}$, and 6138(7)-151.4(2) $\mathrm{keV} \alpha-\gamma$ coincidences. $^{3}$ The $6052(15)-$ and $6138(10)-\mathrm{keV}$ peaks in

\footnotetext{
${ }^{3}$ No energy uncertainty was stated for these decays in Ref. [47]; the present values originate from the re-evaluation of data performed by the authors of the present study.
}

Fig. 9(b) are in agreement with Refs. [12,47]. The prompt $\alpha(6138)-\gamma(151.5)$ coincidences were also observed in the present study (four events in total, not shown in the present spectra). By comparing their number to the number of the $\alpha(6138)$-Ir $K_{\alpha, \beta}$ x rays (in total seven counts), a $K$-shell conversion coefficient, $\alpha_{K \text {,exp }}(151.5)=1.3(7)$, was deduced, which is within the uncertainty of $\alpha_{K, \text { th }}(M 1,151.5)=1.56$ [35] $\left[\alpha_{K, \mathrm{th}}(E 1,151.5)=0.11, \alpha_{K, \mathrm{th}}(E 2,151.5)=0.34\right]$. This tentatively establishes an $M 1$ (or possibly, mixed $M 1 / E 2$ ) multipolarity for the $151.5-\mathrm{keV}$ transition.

The weak 5798- and 6157-keV decays seen in Ref. [12] were not observed in the present work due to the lower statistics collected, but we assume their presence and added them to the decay scheme in Fig. 10(b). The intensities of all five decays of ${ }^{176} \mathrm{Au}^{\text {ls }}$ were recalculated by accounting for the measured data for 6052- and 6138-keV decays in Fig. 9(b), and for the intensities for the 5798- and 6157-keV decays taken from Ref. [12].

The relatively low statistics collected for ${ }^{176} \mathrm{Au}$ prevented us from using the grow-in/decay method for the half-life determination of both states. Due to this, the half-life values from Ref. [9] are used in the decay scheme in Fig. 10.

\section{3. ${ }^{176} \mathrm{Au}^{\mathrm{ls}, \mathrm{hs}} \alpha$-decay branching ratios}

The only previously reported $\alpha$-decay branching ratio $b_{\alpha}\left({ }^{176} \mathrm{Au}^{\mathrm{ls}}\right)=75(8) \%$ is from Ref. [51], although the method of calculation is not divulged.

In our study, the $b_{\alpha}$ values for both states were derived by comparing the number of $\alpha$ decays from ${ }^{176} \mathrm{Au}^{\mathrm{ls} \text {, hs }}$ and their $\beta$-decay daughter ${ }^{176} \mathrm{Pt}$ in Figs. 9(a) and 9(b). For example, $b_{\alpha}\left({ }^{176} \mathrm{Au}^{\mathrm{ls}}\right)$ can be derived by using the expression in Eq. (1) (a similar expression is also used for the high-spin isomer):

$$
\begin{aligned}
b_{\alpha}\left({ }^{176} \mathrm{Au}\right) & =\frac{N_{\alpha}\left({ }^{176} \mathrm{Au}^{\mathrm{ls}}\right)}{N_{\mathrm{tot}}\left({ }^{176} \mathrm{Au}^{\mathrm{ls}}\right)}=\frac{N_{\alpha}\left({ }^{176} \mathrm{Au}^{\mathrm{ls}}\right)}{N_{\alpha}\left({ }^{176} \mathrm{Au}^{\mathrm{ls}}\right)+N_{\text {tot }}\left({ }^{176} \mathrm{Pt}\right)} \\
& =\frac{N_{\alpha}\left({ }^{176} \mathrm{Au}^{\mathrm{ls}}\right)}{N_{\alpha}\left({ }^{176} \mathrm{Au}{ }^{\mathrm{ls}}\right)+\frac{\left.N_{\alpha}{ }^{176} \mathrm{Pt}\right)}{b_{\alpha}\left({ }^{176} \mathrm{Pt}\right)}},
\end{aligned}
$$

where $b_{\alpha}\left({ }^{176} \mathrm{Pt}\right)=40(2) \%$ is a weighted average from ENSDF [52] and $N_{\text {tot }}$ is the total number of a given isotope produced during the experiment.

Due to the quasirefractory nature of platinum its direct production and extraction from the ion source is strongly suppressed in our experiment. This was demonstrated when the lasers were tuned off the gold ionization scheme and no platinum $\alpha$ decays were observed. Thus, all the ${ }^{176} \mathrm{Pt}$ present in the spectra can only originate from the $\beta^{+} / \mathrm{EC}$ decay of implanted ${ }^{176} \mathrm{Au}^{\text {ls, hs }}$.

No additional corrections were needed in this case, as the wheel of the windmill was only turned every three to four supercycles (108-144 s) of the PS booster, and thus one can neglect a very small removal of daughter activity by the movement of the wheel. This is due to the short half-lives of both isomers of ${ }^{176} \mathrm{Au}(\approx 1.1-1.3 \mathrm{~s})$ and a relatively short half-life of ${ }^{176} \mathrm{Pt}(6.33(15) \mathrm{s}$ [52]).

By direct application of Eq. (1) we deduced the branching ratios as $b_{\alpha}\left({ }^{176} \mathrm{Au}^{\mathrm{ls}}\right)=58(5) \%$ and $b_{\alpha}\left({ }^{176} \mathrm{Au}^{\mathrm{hs}}\right)=29(5) \%$, the latter represents the first determination of this value. Our 


\section{(a) ${ }^{176} \mathrm{Au}{ }^{\mathrm{hs}}$}

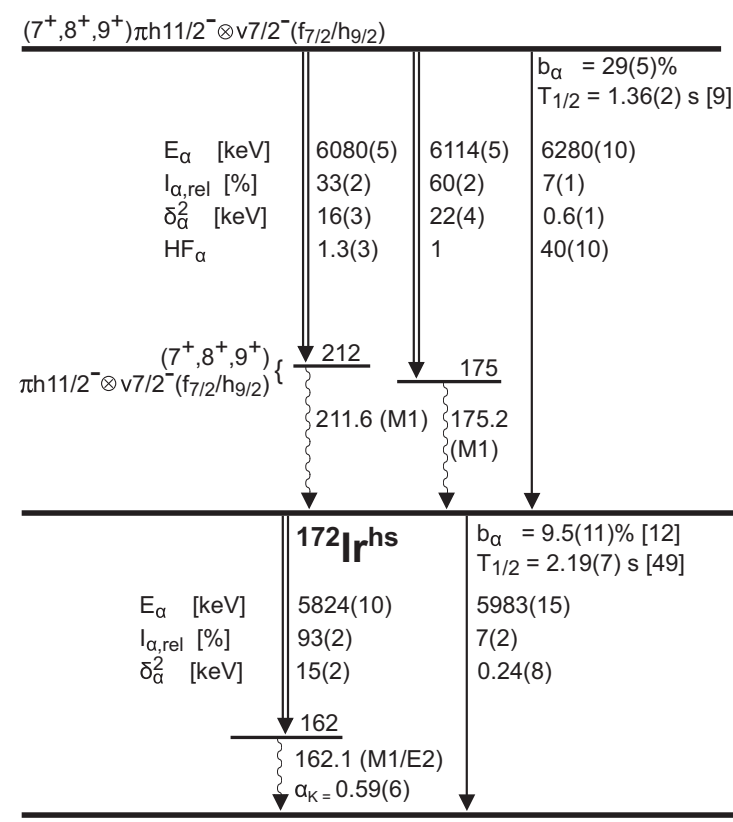

${ }^{168} \operatorname{Re}^{\text {hs }}$ (b) ${ }^{176} \mathrm{Au}^{\text {ls }}$

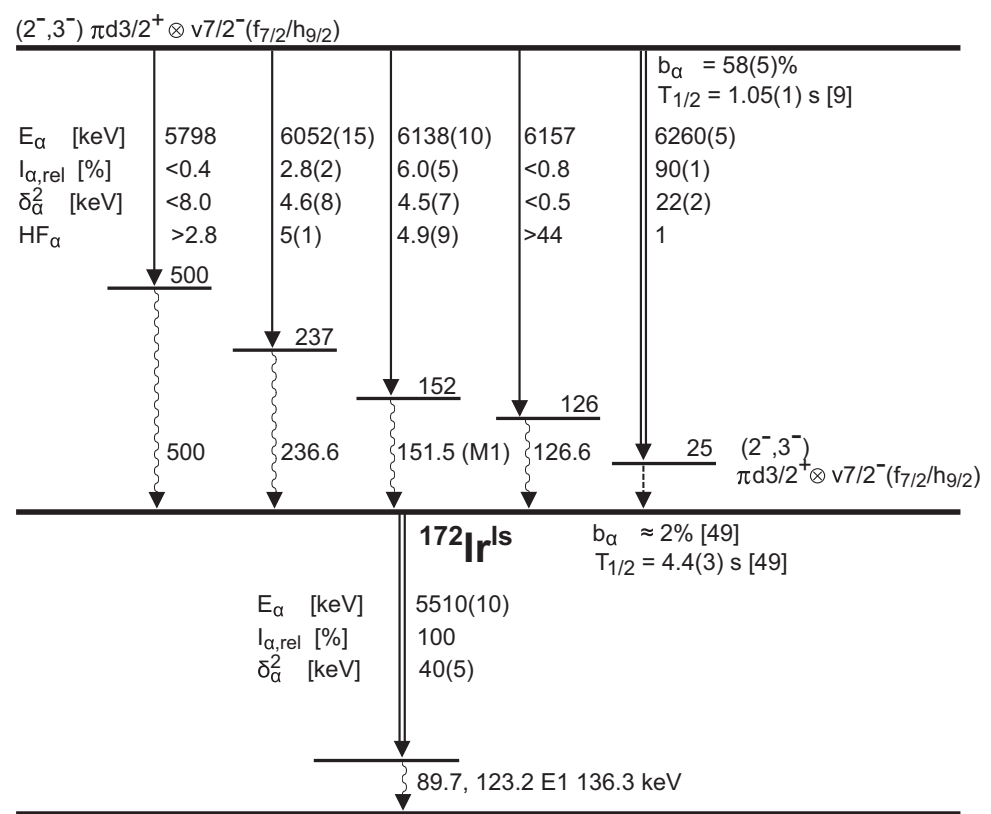

${ }^{168} \operatorname{Re}^{\text {ls }}$

FIG. 10. Decay schemes for ${ }^{176} \mathrm{Au}^{\text {hs }}$ (a) and for ${ }^{176} \mathrm{Au}^{\text {ls }}$ (b). For each $\alpha$-decay branch, the $\alpha$-decay energies $E_{\alpha}$, relative intensities $I_{\alpha \text {,rel }}$, and reduced widths $\delta_{\alpha}^{2}$, calculated using the Rasmussen approach [29], are shown. The values taken from literature are labeled with respective references in square brackets, e.g., evaluated data in Ref. [49] for the high- and low-spin states in ${ }^{172}$ Ir. The 5798- and 6157-keV decays of ${ }^{176} \mathrm{Au}^{\text {ls }}$ were not observed in this study; their existence was assumed from Ref. [12], with the relative intensives for f.s. decays of ${ }^{176} \mathrm{Au}{ }^{\text {ls }}$ derived as explained in Sec. III C 2. The energies for $\gamma$ rays from excited states in ${ }^{172} \mathrm{Ir}$ are from the SHIP study [12], except for the 500.3(3)and 475.4(5)-keV decay, taken from Ref. [47]. The excitation energy of the 25-keV state was deduced from the energy difference of the 500.3and 475.4-keV $\gamma$ rays; see Sec. IV C 3 for discussion of this procedure.

branching value for the ls state is within $\approx 3 \sigma$ limit from the value from Ref. [51].

\section{DISCUSSION}

\section{A. ${ }^{179} \mathrm{Au}$}

To start the discussion of the decay scheme of ${ }^{179} \mathrm{Au}$ in Fig. 3, it is important to mention that no evidence for a f.s. decay with an energy of $\approx 5820 \mathrm{keV}$ to the $26.1-\mathrm{keV}\left(3 / 2^{+}\right)$ excited state in ${ }^{175} \mathrm{Ir}$ has been seen in our data. The strong internal conversion of the respective $26.1-\mathrm{keV} M 1$ transition $\left(\alpha_{\text {tot,exp }}=76(14)\right.$ [28]) would lead to the appearance of lowenergy conversion electrons, with the energies of $\approx 12 \mathrm{keV}$ ( $L$-shell conversion) and $\approx 23 \mathrm{keV}(M$-shell), with an intensity ratio of $L / M \approx 4.3$. The respective $\alpha(5820)+\mathrm{e}^{-}(L / M)$ summing in the silicon detectors of WM could give rise to an artificial peak with an energy $\approx 5-15 \mathrm{keV}$ lower than the main 5848-keV decay. This scenario was checked with GEANT4 simulations, which included both $L$ - and $M$-shell conversion; no visible effect on the simulated shape of the 5848-keV line was noted. In agreement with this, the experimental FWHM for the $5848-\mathrm{keV}$ peak was found to be the same as for the single $\alpha$-decay peaks for other isotopes studied in this experiment. Furthermore, none of the previous studies have mentioned any possible issues in respect of the shape or width of the 5848-keV peak.
Due to this, we assume that the $5820-\mathrm{keV}$ decay either does not exist, or it has a negligible intensity in comparison to the 5848-keV decay. Under these assumptions, and relying on so far a single measurement of $b_{\alpha}\left({ }^{179} \mathrm{Au}\right)=22.0(9) \%$ from Ref. [27], a reduced $\alpha$-decay width of $\delta_{\alpha}^{2}(5848 \mathrm{keV})$ $=58(4) \mathrm{keV}$ was derived. This value is within the typical range for unhindered $\alpha$ decays in this region, which is seen from its comparison to, e.g., neighboring even-even isotopes ${ }_{178,180,182} \mathrm{Hg}$, having $\delta_{\alpha}^{2} \approx 55-70 \mathrm{keV}$; see Fig. 11. Based on this fact and on the assignment of $I^{\pi}\left({ }^{179} \mathrm{Au}^{\mathrm{g}}\right)=1 / 2^{+}$from Ref. [15], the state in the daughter nucleus ${ }^{175} \mathrm{Ir}$, populated by the 5848-keV decay, should also be assigned as $I^{\pi}=\left(1 / 2^{+}\right)$. Actually, this argument has already been used in the recent investigation of ${ }^{175} \mathrm{Ir}$ in Ref. [28], with a reference to the $\alpha$-decay data from the present study.

The 5718-, 5705-, and 5600-keV $\alpha$ decays are quite strongly hindered, by at least a factor of $\approx 22$ or more, relative to the 5848-keV transition; see Fig. 3. However, due to the lack of further data, no conclusions can be drawn on the nature of the respective excited states in ${ }^{175} \mathrm{Ir}$, fed by these decays.

The unhindered character of the $1 / 2^{-} \rightarrow\left(1 / 2^{-}\right)$, $5188-\mathrm{keV}$ decay of ${ }^{179} \mathrm{Pt}$ was known from the previous studies; it presumably feeds the $102.3-\mathrm{keV}, 1 / 2^{-}$[521] bandhead of the known rotational band in ${ }^{175}$ Os. The f.s. $5116-\mathrm{keV}$ decay identified in the present investigation is 


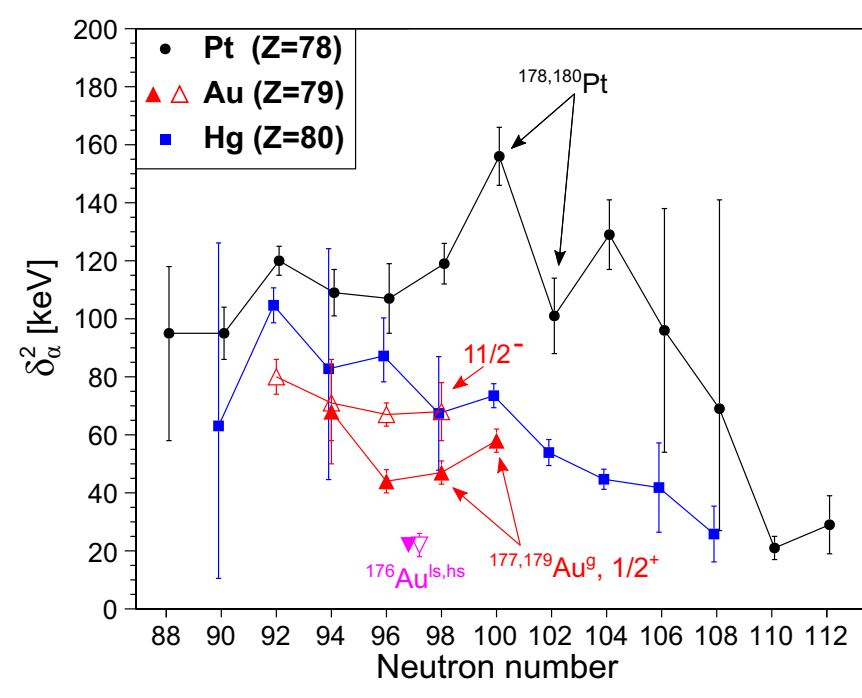

FIG. 11. A comparison of the reduced $\alpha$-decay widths for g.s. $\rightarrow$ g.s. decays of even-even platinum and mercury isotopes with the data for ${ }^{171,173,175,176,177,179} \mathrm{Au}$. All values were calculated using the Rasmussen approach [29], with literature data taken from ENSDF. The open and closed red triangles represent the decays of isomeric $11 / 2^{-}$and $1 / 2^{+}$ground states in the odd- $A$ gold isotopes, respectively. The new data for ${ }^{176} \mathrm{Au}^{\text {ls, hs }} \mathrm{Au}$ from this study are shown by the pink triangles.

proposed to feed the known $\left(3 / 2^{-}\right)$member of the same band at $175.6 \mathrm{keV}$; see Fig. 3. This decay is only weakly hindered, by a factor of $\approx 2$, relative to the $5188-\mathrm{keV}$ decay, which could be due to its $\Delta L=2$ character. This reflects a typical trend in this region of nuclei, when the decays to the states of the same band are weakly and similarly hindered. For example, in the ${ }^{181} \mathrm{Hg} \rightarrow{ }^{177} \mathrm{Pt}$ case, the $1 / 2^{-} \rightarrow 1 / 2^{-} \alpha$ decay is unhindered $\left[H F_{\alpha}=0.86(12)\right]$, while the two f.s. decays to the $\left(3 / 2^{-}\right)$and $5 / 2^{-}$excited states in ${ }^{177} \mathrm{Pt}$ have moderate and comparable hindrance factors of 6.5(20) and 7(3), respectively.

It is interesting to note that the sum of the reduced widths for the 5116- and 5188-keV decays adds up to $\approx 120 \mathrm{keV}$, which is comparable to the average of the two even-even neighbors ${ }^{178,180} \mathrm{Pt}$; see Fig. 11 . In other words, the total $\alpha$ decay strength of ${ }^{179} \mathrm{Pt}$ seems to be practically the same as of ${ }^{178,180} \mathrm{Pt}$.

\section{B. ${ }^{177} \mathrm{Au}$}

Apart from confirming the previously known $\alpha$-decay energies for the strongest decays of ${ }^{177} \mathrm{Au}^{\mathrm{g}, \mathrm{m}}$ (see Fig. 8), a new f.s. $\alpha$ decay at $5932 \mathrm{keV}$ was found for ${ }^{177} \mathrm{Au}^{\mathrm{m}}$.

The recent $g$-factor measurement for the isomeric $I^{\pi}\left({ }^{177} \mathrm{Au}^{\mathrm{m}}\right)=11 / 2^{-}$state supported its most likely configuration as $\pi 11 / 2^{-}[505] \mathrm{h}_{11 / 2}$, similar to such isomeric states in the heavier odd- $A \mathrm{Au}$ isotopes; see Ref. [18]. Thus, the unhindered character of its $6125-\mathrm{keV}$ decay confirms a similar configuration for the daughter $\left(11 / 2^{-}\right)$state in ${ }^{173} \mathrm{Ir}$. We note a near constancy of the $\delta_{\alpha}^{2}\left(11 / 2^{-}\right)$values at $\approx 70-80 \mathrm{keV}$ in ${ }^{171,173,175,177} \mathrm{Au}^{\mathrm{m}}$; see Fig. 11.
In contrast, the $11 / 2^{-} \rightarrow\left(9 / 2^{-}\right) 5932-\mathrm{keV}$ decay is hindered by a factor of $\approx 12$ relative to the $6125-\mathrm{keV}$ decay of ${ }^{177} \mathrm{Au}^{\mathrm{m}}$. The hindrance is mostly likely caused by its $\Delta L=2$ character combined with a change of the proton component of the daughter $\left(9 / 2^{-}\right)$state, proposed as being the lowest state of the $\pi 1 / 2^{-}[541] h_{9 / 2}$ configuration [40].

The $6156-\mathrm{keV}$ decay of the $I^{\pi}\left({ }^{177} \mathrm{Au}^{\mathrm{g}}\right)=1 / 2^{+}$state has a reduced $\alpha$-decay width of $47(4) \mathrm{keV}$, which is comparable to the respective values for ${ }^{175,179} \mathrm{Au}^{\mathrm{g}}$ (see Fig. 11). A rather similar structure of ${ }^{177,179} \mathrm{Au}^{\mathrm{g}}$ was also confirmed by a similarity of their $g$ factors in Ref. [15].

The study [42] tentatively assigned a spin/configuration of $\left(3 / 2^{+}[402]\right)$ or $\left(5 / 2^{+}\right.$[512]) for ${ }^{173} \mathrm{Ir}^{\mathrm{g}}$, which was based on its $\beta$-decay feeding pattern to the states in ${ }^{173}$ Os and also on the systematics of the states in the Ir isotopes in this region; see Fig. 9 in their paper. A latter investigation of the $\alpha$-decay chain ${ }^{177} \mathrm{Au}^{\mathrm{g}} \rightarrow{ }^{173} \mathrm{Ir}^{\mathrm{g}} \rightarrow{ }^{169} \mathrm{Re}$ in Ref. [9] proposed a spin and parity of $\left(1 / 2^{+}, 3 / 2^{+}\right)$to the low-spin state in ${ }^{173} \mathrm{Ir}$, partially based on the $\left(1 / 2^{+}, 3 / 2^{+}\right)$assignment for ${ }^{177} \mathrm{Au}^{\mathrm{g}}$ known at that time; see also the discussions in Refs. [8,10,38]. Based both on $I^{\pi}\left({ }^{177} \mathrm{Au}^{\mathrm{g}}\right)=1 / 2^{+}$from Ref. [15] and on the unhindered or weakly hindered character of the $6156-\mathrm{keV}$ decay, we confirm an $\left(1 / 2^{+}, 3 / 2^{+}\right)$assignment for ${ }^{173} \mathrm{Ir}^{\mathrm{g}}$ but rule out $\left(5 / 2^{+}\right)$suggested in Ref. [42].

$$
\text { C. }{ }^{176} \mathrm{Au}
$$

\section{Magnetic moment determination for ${ }^{176} \mathrm{Au}^{\mathrm{ls}, \mathrm{hs}}$}

The previous studies of ${ }^{176} \mathrm{Au}^{\text {ls, hs }}[9,12]$ relied mostly on $\alpha$-decay systematics in this region for the tentative spin and parity assignments for both states. The hfs data in Fig. 6(b) provide an opportunity to test these inferences, via the determination of magnetic moments for these states. The latter might also help determine the underlying proton-neutron configurations. To illustrate the analysis procedures, we discuss below the case of the high-spin state in ${ }^{176} \mathrm{Au}$; the low-spin data were treated in the same way.

The positions of the hyperfine components as a function of the scanning laser frequency in Fig. 6(b) are determined by the expression

$$
v_{F, F^{\prime}}=v_{0}+a(6 p) \frac{K^{\prime}}{2}-a(6 s) \frac{K}{2},
$$

where $v_{0}$ is the centroid frequency of the hfs, the primed and un-primed symbols denote the upper $6 p{ }^{2} P_{1 / 2}$ atomic state, and $6 s^{2} S_{1 / 2}$ ground states, respectively, connected by the 267.6-nm atomic transition, $K=F(F+1)-I(I+1)-$ $J(J+1)$, and $a(n l)$ is the magnetic hyperfine coupling constant for the atomic level with the quantum numbers $n$ and $l$.

The hfs spectra for ${ }^{176} \mathrm{Au}^{\text {ls, hs }}$ in Fig. 6(b) were fitted using Voigt profiles, with a range of spin assignments, which was limited by the previous decay studies $[9,12,46]: I=7-10$ and $2-5$ for the hs and ls states, respectively. Specifically, the $\beta$-decay study [46] suggested the most probable spin assignments of 2 and 8 for ${ }^{176} \mathrm{Au}^{\text {ls, hs }}$. The hfs patterns for ${ }^{176} \mathrm{Au}^{\mathrm{ls}, \mathrm{hs}}$ can be equally well reproduced by the fits with any of the respective spin assignments, and therefore only the fits with $I=3$ and 9 are shown by the red line in Fig. 6(b). For each 
spin assumption, the deduced values of $a(6 s)$ and ratios of $a(6 p) / a(6 s)$ are given in Table II.

To determine the magnetic dipole moments the standard relation is used:

$$
\mu=\mu_{\text {ref }} \frac{I_{A}}{I_{\text {ref }}} \frac{a_{A}}{a_{\text {ref }}}\left(1+{ }^{\text {ref }} \Delta^{A}\right),
$$

where the subscript (superscript) "ref" denotes a reference isotope $\left({ }^{197} \mathrm{Au}\right)$ with known $\mu$ and $a$ values, and ${ }^{\text {ref }} \Delta^{A}$ is a relative hyperfine anomaly (RHFA) stemming from the nonpoint-like charge and magnetization distribution inside the nucleus; see, e.g., Refs. $[53,54]$ and references therein.

Usually, the prescription by Ekström et al. [53] is used to account for large RHFA in gold isotopes when calculating their magnetic moments:

$$
\mu=\frac{a(6 s) I}{29005} \mu_{N}
$$

However, it has recently been shown in Ref. [18] that this prescription should be reconsidered. As suggested in Ref. [18], the RHFA values for the gold isotopes can be directly deduced, based on advanced atomic calculations and from the ratio of the magnetic hfs constants for the $6 p$ and $6 s$ atomic states. Applying this procedure, we obtain the values of ${ }^{197} \Delta{ }^{176 \mathrm{hs}}$ for different spin assumptions; see Table II.

By using values of $a_{6 s}\left({ }^{197} \mathrm{Au}\right)=3049.660092(7) \mathrm{MHz}$ $[55], a_{6 p}\left({ }^{197} \mathrm{Au}\right)=312.7(1.2) \mathrm{MHz}$ [5], and $\mu\left({ }^{197} \mathrm{Au}\right)=$ $0.14574(4) \mu_{N}$ from Ref. [55], and with the diamagnetic correction from Ref. [56], we obtain by Eq. (3) the magnetic moments for different spin assignments for ${ }^{176} \mathrm{Au}^{\text {hs }}$ which include the RHFA corrections; see Table II. This table also shows the experimental magnetic moments deduced with the Ekström et al. prescription [Eq. (4)], with revised uncertainties according to Ref. [18].

\section{Possible spin and configurations for ${ }^{176} \mathrm{Au}^{\mathrm{ls}, \mathrm{hs}}$}

A choice of the most likely spins and configurations for ${ }^{176} \mathrm{Au}^{\text {ls,hs }}$ was made by comparing their experimental $\mu$ values with the semiempirical values for odd-odd configurations calculated using a well-known additivity expression for proton-neutron coupling [57]. Both isomers in ${ }^{176} \mathrm{Au}$ are nearly spherical, which will be discussed elsewhere [58], and therefore we used the standard additivity relation, which works well for spherical and weakly deformed nuclei [59].

In additivity-relation calculations the magnetic moments of the adjacent odd- $A$ nuclei were used as the single-particle values. The most probable proton orbitals involved in ${ }^{176} \mathrm{Au}$ are $\pi h_{11 / 2}, \pi s_{1 / 2}$, and $\pi d_{3 / 2}$, as they are known to be responsible for the spins of the low-lying isomeric $11 / 2^{-}$and $1 / 2^{+}$ ground states in the neighboring odd- $A$ isotopes ${ }^{175,177} \mathrm{Au}$; see Figs. 8 and 3 of [12]. In the case of the $d_{3 / 2}$ proton state, a mean value of the magnetic moments of the nearly spherical $I=3 / 2^{+}$ground states in ${ }^{193,195,197}$ Au was used.

The neutron configuration should involve $v f_{7 / 2}$ and/or $v h_{9 / 2}$ orbitals, which are known to determine the spins of the odd- $N$ isotopes in the region of the neutron numbers $N=95-99$. The known magnetic moment of the $I=7 / 2^{-}$ ground state in ${ }^{177} \mathrm{Hg}$ [60] was used for the $v f_{7 / 2}$ orbital, while an estimation from Ref. [61] was employed for the $v h_{9 / 2}$ orbital.

The results of the additivity calculations for several protonneutron configurations, leading to the nuclear spins within the limitations established by the nuclear spectroscopic decay studies, are compared with the experimental data in Table III.

For the high-spin isomer, a good agreement between the experimental and semiempirical magnetic moments is seen for the $\left[\pi h_{11 / 2} \otimes v f_{7 / 2}\right]_{7^{+}, 8^{+}, 9^{+}}$and $\left[\pi h_{11 / 2} \otimes v h_{9 / 2}\right]_{7^{+}}$configurations. The predominant $\beta$-decay feeding the $8^{+}$excited state in the daughter ${ }^{176} \mathrm{Pt}$, in comparison with, e.g., feeding the $6^{+}$or $10^{+}$states (see Fig. 7 of [46]), supports any of the $I^{\pi}=7^{+}-9^{+}$spin assignments. Due to this, we prefer to keep all of them in the decay scheme of ${ }^{176} \mathrm{Au}^{\text {hs }}$.

For the low-spin isomer, only a $\left[\pi d_{3 / 2} \otimes \nu f_{7 / 2}\right]_{2^{-}, 3^{-}, 4^{-}, 5^{-}}$ configuration provides a good agreement, both in respect of the sign and the value, between the experimental and semiempirical magnetic moments. The somewhat worse agreement for the $2^{-}$assumption might be due to possible deficiencies with the choice of the single-particle magnetic moments for the additivity calculations. A strong $\beta$-decay feeding the $2^{+}$ excited state in the daughter ${ }^{176} \mathrm{Pt}$ (see Fig. 7 of Ref. [46]) supports the preference for the $I^{\pi}=2^{-}$or $3^{-}$spin assignment for ${ }^{176} \mathrm{Au}^{\mathrm{ls} 4}$.

This preferred configuration indicates a change to a pure $d_{3 / 2}$ proton state in the odd-odd ${ }^{176} \mathrm{Au}^{\text {ls }}$ in contrast with the $1 / 2^{+}$ground state of the adjacent ${ }^{177,179} \mathrm{Au}^{\mathrm{m}}$ where a strong mixing of the $d_{3 / 2}$ and $s_{1 / 2}$ proton states was observed [15].

Remarkably, an odd neutron occupies the $f_{7 / 2}$ shell in both ${ }^{176} \mathrm{Au}^{\text {ls,hs. }}$. This assignment testifies that the "shell swap" (change in the order of $v f_{7 / 2}$ and $v h_{9 / 2}$ orbitals) found earlier for the ${ }^{177,179} \mathrm{Hg}^{\mathrm{m}}$ isotopes $(Z=80, N=97,99)$, is also preserved for $Z=79, N=97$, whereas it disappears for isotonic lead and thallium nuclei $(Z=82,81)$; see Ref. [60] and references therein.

\section{3. $\alpha$-decay properties of ${ }^{176} \mathrm{Au}$}

${ }^{176} \mathrm{Au}^{\text {hs }}$ decay chain. As mentioned earlier, the present study confirmed most of the conclusions from Ref. [12] for ${ }^{176} \mathrm{Au}^{\text {hs }}$ in terms of the $\alpha$-decay energies and relative intensities. However, the first experimental determination of $b_{\alpha}\left({ }^{176} \mathrm{Au}^{\mathrm{hs}}\right)=29(5) \%$ in the present investigation leads to a reduction in the reduced $\alpha$-decay widths for the $\alpha$ decays of the high-spin isomer by a factor of $\approx 3.3$, as a $100 \%$ branching ratio was previously assumed in Ref. [12]. Due to this, the 6080- and 6114-keV decays have now values of $\delta_{\alpha}^{2} \approx 16-22$ $\mathrm{keV}$, which are $\approx 4$ times lower than the reduced widths of $\approx 70-80 \mathrm{keV}$ for the unhindered $\alpha$ decays of the $11 / 2^{-}$isomeric states in the neighboring ${ }^{175,177} \mathrm{Au}$; see Fig. 11. To keep a meaningful comparison, we only employ here the decays from the proton $h_{11 / 2}$-based states in ${ }^{175,176,177} \mathrm{Au}$.

However, at least a part of the reduction of $\alpha$-decay reduced widths in odd-odd isotopes versus their odd- $A$ neighbors (or

\footnotetext{
${ }^{4}$ Based on the preliminary analysis from present laser-spectroscopy data, the $\left[\pi d_{3 / 2} \otimes v f_{7 / 2}\right]_{2^{-}, 3^{-}, 4^{-}, 5^{-}}$configuration was already suggested by our collaboration in Ref. [47], but we kept the spin assignment of 4,5 from the SHIP study [12] at the time.
} 
TABLE III. Possible neutron-proton configurations for ${ }^{176} \mathrm{Au}^{\mathrm{ls}, \mathrm{ss}}$ leading to the nuclear spins, $I$, limited by the available nuclear spectroscopic decay data; see the text for details. The magnetic dipole moment, $\mu_{\text {add }}$, of each configuration was calculated using the spherical additivity relations. The last two columns show the magnetic moments deduced in this study within the Ekström et al. approach [53] and with the RHFA correction following the prescription from Ref. [18], respectively. The preferred configurations are shown in bold. Further $p-n$ configurations are possible, but they are not shown in the table as they could be ruled out by large deviations of calculated absolute values and/or signs of their magnetic moments in comparison to experimental data.

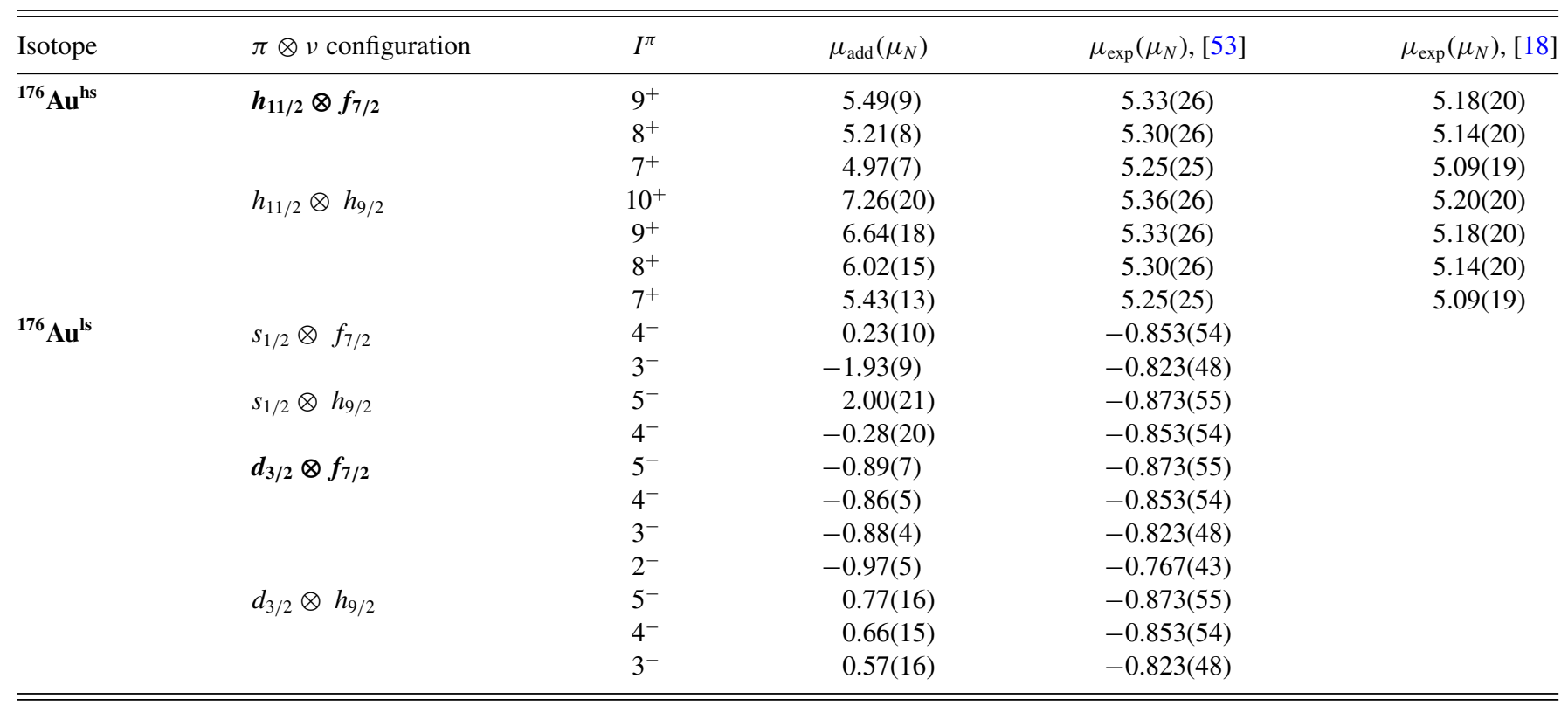

odd- $A$ versus even-even neighbors) is usually attributed to the so-called blocking effect in the $\alpha$-particle formation. In the simplest case, an odd nucleon serves as an "observer" in an odd- $A$ nucleus and just blocks an orbital which was previously available for pairing and $\alpha$-particle formation in the even-even neighbor, without a substantial change in the wave function of the core nucleus. As one example, we refer the reader to the discussion of the spin-dependent blocking of the neutron $i_{13 / 2}$ and $p_{3 / 2}$ orbitals in the $\alpha$ decay of ${ }^{193-197}$ Po in Ref. [62]. As shown in Table I of Ref. [62], a typical hindrance factor of $\approx 2-3$ was observed for these $\alpha$ decays relative to the decays in the even-even Po neighbors. Even large hindrance could be expected for an odd-odd nucleus, where both proton and neutron blocking will occur. Based on the above arguments, we consider the 6080- and 6114-keV decays as unhindered, or only weakly hindered, in respect to the unhindered $\alpha$ decays of ${ }^{175,177} \mathrm{Au}^{\mathrm{hs}}$.

Therefore, in Fig. 10 we show the $H F_{\alpha}$ values for the 6080- and 6280-keV decays relative to the strongest 6114-keV decay, for which $H F_{\alpha}=1$ was assumed. Only a small difference in the hindrance of these decays signifies a similarity of the daughter states, which most probably originate from the same proton-neutron multiplet, as involved in the parent $\alpha$ decaying state. On these grounds, we assigned the same range of possible spins of $I=\left(7^{+}, 8^{+}, 9^{+}\right)$to these two states as for ${ }^{176} \mathrm{Au}^{\text {hs }}$ itself. On the other hand, the $6280-\mathrm{keV}$ decay is hindered by a factor of 40(10), and thus the configuration of the $\alpha$-decaying high-spin state in ${ }^{172}$ Ir should be different.

To summarize the discussion of the decay of ${ }^{176} \mathrm{Au}^{\text {hs }}$, the present study confirmed most of the data and conclusions derived in Ref. [12]. Furthermore, based on the first determination of its magnetic moment and $\alpha$-decay branching ratio, a further clarification on the underlying configurations involved in the $\alpha$ decay of this state was obtained.

${ }^{176} \mathrm{Au}^{\mathrm{ls}}$ decay chain. In contrast to ${ }^{176} \mathrm{Au}^{\mathrm{hs}}$, based on our new data, the decay scheme of ${ }^{176} \mathrm{Au}^{\text {ls }}$ should be crucially modified to account for the observation of the strong 6260$\mathrm{keV}$ decay instead of the 6287 - or $6282-\mathrm{keV}$ decays suggested by studies $[9,12]$.

The four groups of $\alpha-\gamma$ coincidences, observed at 5798$500,6054-236.6,6138-151.5$, and 6157-126 keV in Ref. [12] (first three of them confirmed in Ref. [47]), have the same value of $Q_{\alpha \text {,tot }}=6432(10) \mathrm{keV}$. Importantly, an additional coincidence pair of 5806(10)-475.4(5) keV was seen in Fig. 1(b) of Ref. [47], whose $\alpha$-decay energy matches to the 5798(10)500.3(3) keV coincidence group. Due to this, the study [47] tentatively suggested that the 500.3- and 475.4-keV decays are in coincidence with the same f.s. 5798-keV $\alpha$ decay, and there should be a cascade of 475.4-25 keV transitions, in parallel to the 500-keV decay. The energy difference between the two $\gamma$ rays is $24.9(6) \mathrm{keV}$, but this decay was not observed in Ref. [47], due to its energy being below the registration threshold for $\gamma$ rays in that experiment. Furthermore, the order of the 475.4-24.9 transitions could not be established in Ref. [47]. On the other hand, the "missing" $25-\mathrm{keV}$ decay matches well to the energy difference of $Q_{\alpha \text {,tot }}-Q_{\alpha}(6260)=27(11) \mathrm{keV}$. This fact uniquely establishes that the 6260-keV decay feeds the 24.9-keV state in ${ }^{172} \mathrm{Ir}$, as shown in Fig. 10(b).

The reduced $\alpha$-decay width of 22(2) keV for this transition is lower by a factor of $\approx 3-4$ relative to respective values for the $\alpha$ decays of the $1 / 2^{+}$states in the neighboring ${ }^{175,177} \mathrm{Au}$; see Fig. 11. However, similar to the discussion of the 6114-keV decay of the high-spin state, and by accounting for 
the possible blocking effect for $\alpha$ decay in odd-odd nuclei, the $6260-\mathrm{keV}$ decay is also considered as unhindered or only weakly hindered. Therefore, the spin and underlying configuration of the $25-\mathrm{keV}$ excited state in ${ }^{172} \mathrm{Ir}^{1 \mathrm{~s}}$ should be the same as of the parent state in ${ }^{176} \mathrm{Au}^{\text {ls }}$. Due to this, it was tentatively assigned as having a spin and a parity of $I^{\pi}=\left(2^{-}, 3^{-}\right)$.

The remaining four f.s. decays of ${ }^{176} \mathrm{Au}^{\text {ls }}$ have different degrees of hindrance, as shown in Fig. 10, with quite similar and relatively low hindrance factors for the 6052- and 6138-keV decays. The latter most probably signifies the similar structure for the 152- and 237-keV excited states in ${ }^{172} \mathrm{Ir}^{\mathrm{ls}}$ and a possibility that the 24.9-, 152- and 237-keV states are members of the same $p-n$ multiplet. In contrast, the large hindrance for the 6157-keV decay suggests a very different structure for the 126-keV excited state.

We now turn to the possible decay of the new excited state at $24.9-\mathrm{keV}$ in ${ }^{172} \mathrm{Ir}^{\mathrm{ls}}$. Irrespective of its multipolarity, the corresponding $\gamma$-ray transition will be strongly converted. The resulting conversion electrons from the predominant $L$-shell conversion will have an energy of $\approx 12 \mathrm{keV}$ (or $\approx 22 \mathrm{keV}$ for the $M$-shell conversion). If the $24.9-\mathrm{keV}$ transition is prompt, then the resulting $\alpha(6260)+e^{-}$summing in the silicon detectors of the WM could produce an artificial summing peak with an energy of $\approx 6272-6282 \mathrm{keV}$.

To assess the presence of this decay, we shifted the single $5753-\mathrm{keV}$ peak of ${ }^{176} \mathrm{Pt}$ to the position of the $6260-\mathrm{keV}$ peak, see Fig. 9(b), and also normalized them to the same intensity. A small excess of events on the right-hand side of the $6260-\mathrm{keV}$ peak can be seen with respect of the shifted 5753 $\mathrm{keV}$ peak. Albeit very tentatively, this excess can be ascribed to the above-mentioned $\alpha(6260)+e^{-}$summing. As stated earlier, the probability of $\alpha+e^{-}$summing is relatively low in the WM geometry, as the activity is implanted in an external carbon foil, which reduces the chance of both $\alpha$ particle and conversion electron to be simultaneously registered in the same detector.

In contrast, in the studies [9,12], which employed complete-fusion reactions at recoil separators, the activity was directly implanted in a silicon detector at a depth of several microns, and thus the probability of $\alpha+e^{-}$summing becomes high, especially for low-energy electrons. In our opinion, this is the reason for the observation of the 6280 - or $6287-\mathrm{keV}$ peaks in these experiments, whereby the full-energy $\alpha+e^{-}$ summing was most probably observed. The above considerations also require that the $24.9-\mathrm{keV}$ transition is prompt, thus being of $E 1$ or $M 1$ character, as any other multipolarity would result in a delayed decay, leading to no $\alpha+e^{-}$ summing.

\section{Decay of the daughter isotope ${ }^{172} \mathrm{Ir}$}

Based on a variety of experimental techniques, several earlier studies suggested a number of possible spin and configuration assignments for the high- and low-spin states in ${ }^{172} \mathrm{Ir}$; see the evaluation in Ref. [49]. In particular, for the high-spin state, assignments of $I=\left(7^{+}\right)$were proposed in Refs. [12,42], $\left(8^{+}\right)$in Ref. [9], and $(5,6)$ in Ref. [63]. For the low-spin state, values of $I=\left(3^{+}\right)$were proposed in Ref. [42], $\left(3^{-}\right)$in Ref. [9], $\left(3^{-}, 4^{-}\right)$in Ref. [12], and $(2,3)$ in Ref. [63].

However, none of the above methods could provide an unambiguous spin or configuration assignment, and they often relied on a complementary use of experimental systematics, e.g., on the $\beta$-decay pattern in Refs. $[42,63]$ or $\alpha$-decay systematics in Refs. [9,12,42].

In the absence of conclusive data, we refrain from speculations on the spins and configurations of the long-lived states in

${ }^{172}$ Ir. Therefore, we do not provide them in the decay schemes in Fig. 10. Further experiments are needed, in particular employing the laser spectroscopy technique, which can provide direct information on spin and electromagnetic moments.

\section{CONCLUSION}

A detailed nuclear spectroscopy study of ${ }^{176,177,179} \mathrm{Au}$ was performed using the RILIS and Windmill decay station at ISOLDE. The use of selective laser ionization coupled with mass separation allowed the production of the isomerically separated beams of the high- and low-spin states in ${ }^{176,177} \mathrm{Au}$. New and improved data on complex fine-structure $\alpha$ decays of the three isotopes were deduced, providing insight into the low-lying levels in the daughter nuclei ${ }^{172,173,175} \mathrm{Ir}$.

The first measurements of the hfs for atomic transitions in ${ }^{176} \mathrm{Au}{ }^{\mathrm{ls}, \mathrm{hs}}$ were also presented, from which the respective magnetic dipole moments have been extracted. The most probable configurations of $\pi d_{3 / 2} \otimes v f_{7 / 2}$ and $\pi h_{11 / 2} \otimes v f_{7 / 2}$ were proposed for the low- and high-spin states, respectively. Thus, the odd neutron occupies the $f_{7 / 2}$ shell in both ${ }^{176} \mathrm{Au}^{\text {ls, hs }}$, which supports the change in the order of $v f_{7 / 2}$ and $v h_{9 / 2}$ orbitals for $Z=79$ and $N=97$, as found earlier for the ${ }^{177,179} \mathrm{Hg}$ isotopes $(Z=80, N=97,99)$.

\section{ACKNOWLEDGMENTS}

We thank K. Blaum, D. Lunney, V. Manea, and L. Schweikhard for their careful reading of the manuscript. We would like to acknowledge the support of the ISOLDE Collaboration and technical teams. We thank the GSI target group for supplying the thin carbon foils used in WM measurements. This work has received funding from the European Union's Horizon 2020 research and innovation programme under Grant Agreement No. 654002 (ENSAR2), and also funding through the European Union's Seventh Framework Programme for Research and Technological Development under Grant Agreements No. 262010 (ENSAR), No. 267194 (COFUND), and No. 289191 (LA3NET). The work was further supported by RFBR according to the Research Project No. 19-02-00005, by the U.K. Science and Technology Facilities Council grants, by the Research Foundation Flanders (FWO, Belgium) Excellence of Science program (EOS, FWO-FNRS, Belgium), by GOA/2015/010 (BOF KU Leuven), by the Interuniversity Attraction Poles Programme initiated by the Belgian Science Policy Office (BriX network P7/12), by the Slovak Research and Development Agency (Contract No. APVV-14-0524), and by the Slovak Grant Agency VEGA (Contract No. 1/0532/17). 
[1] K. Heyde and J. L. Wood, Rev. Mod. Phys. 83, 1467 (2011).

[2] J. L. Wood and K. Heyde, J. Phys. G: Nucl. Part. Phys. 43, 020402 (2016).

[3] U. Krönert, S. Becker, G. Bollen, M. Gerber, T. Hilberath, H. J. Kluge, and G. Passler, Z. Phys. A 331, 521 (1988).

[4] K. Wallmeroth, G. Bollen, A. Dohn, P. Egelhof, U. Krönert, M. J. G. Borge, J. Campos, A. Rodriguez Yunta, K. Heyde, C. De Coster, J. L. Wood, and H.-J. Kluge, Nucl. Phys. A 493, 224 (1989).

[5] G. Passler, J. Rikovska, E. Arnold, H.-J. Kluge, L. Monz, R. Neugart, H. Ravn, and K. Wendt, Nucl. Phys. A 580, 173 (1994).

[6] G. Savard, J. E. Crawford, J. K. Lee, G. Thekkadath, H. T. Duong, J. Pinard, F. Le Blanc, P. Kilcher, J. Obert, J. Oms, J. C. Putaux, B. Roussiere, and J. Sauvage, Nucl. Phys. A 512, 241 (1990).

[7] F. Le Blanc, J. Obert, J. Oms, J. C. Putaux, B. Roussière, J. Sauvage, J. Pinard, L. Cabaret, H. T. Duong, G. Huber, M. Krieg, V. Sebastian, J. Crawford, J. K. P. Lee, J. Genevey, and F. Ibrahim (ISOLDE Collaboration), Phys. Rev. Lett. 79, 2213 (1997).

[8] F. G. Kondev, M. P. Carpenter, R. V. Janssens, K. Abu Saleem, I. Ahmad, H. Amro, J. A. Cizewski, M. Danchev, C. N. Davids, D. J. Hartley, A. Heinz, T. L. Khoo, T. Lauritsen, C. J. Lister, W. C. Ma, G. L. Poli, J. Ressler, W. Reviol, L. L. Riedinger, D. Seweryniak et al., Phys. Lett. B 512, 268 (2001).

[9] J. T. Goon, Alpha and gamma-ray spectroscopic studies of Au, $\mathrm{Pt}$, and Ir nuclei near the proton dripline, Ph.D. thesis, University of Tennessee, Knoxville, 2004.

[10] A. N. Andreyev, S. Antalic, D. Ackermann, T. E. Cocolios, V. F. Comas, J. Elseviers, S. Franchoo, S. Heinz, J. A. Heredia, F. P. Heßberger, S. Hofmann, M. Huyse, J. Khuyagbaatar, I. Kojouharov, B. Kindler, B. Lommel, R. Mann, R. D. Page, S. Rinta-Antila, P. J. Sapple et al., Phys. Rev. C 80, 024302 (2009).

[11] M. Venhart, A. N. Andreyev, J. L. Wood, S. Antalic, L. Bianco, P. T. Greenlees, U. Jakobsson, P. Jones, R. Julin, S. Juutinen, S. Ketelhut, M. Leino, M. Nyman, R. D. Page, P. Peura, P. Rahkila, J. Sarén, C. Scholey, J. Sorri, J. Thomson et al., Phys. Lett. B 695, 82 (2011).

[12] A. N. Andreyev, S. Antalic, D. Ackermann, T. E. Cocolios, J. Elseviers, S. Franchoo, S. Heinz, F. P. Heßberger, S. Hofmann, M. Huyse, J. Khuyagbaatar, B. Kindler, B. Lommel, R. Mann, R. D. Page, P. Van Duppen, and M. Venhart, Phys. Rev. C 90, 044312 (2014).

[13] M. Venhart, F. A. Ali, W. Ryssens, J. L. Wood, D. T. Joss, A. N. Andreyev, K. Auranen, B. Bally, M. Balogh, M. Bender, R. J. Carroll, J. L. Easton, P. T. Greenlees, T. Grahn, P.-H. Heenen, A. Herzáň, U. Jakobsson, R. Julin, S. Juutinen, D. Kíč et al., Phys. Rev. C 95, 061302(R) (2017).

[14] M. Venhart, J. L. Wood, M. Sedlák, M. Balogh, M. Bírová, A. J. Boston, T. E. Cocolios, L. J. Harkness-Brennan, R.-D. Herzberg, L. Holub, D. T. Joss, D. S. Judson, J. Kliman, J. Klimo, L. Krupa, J. Lušnák, L. Makhathini, V. Matoušek, Š. Motyčák, R. D. Page et al., J. Phys. G: Nucl. Part. Phys. 44, 074003 (2017).

[15] J. G. Cubiss, A. E. Barzakh, A. N. Andreyev, M. Al Monthery, N. Althubiti, B. Andel, S. Antalic, D. Atanasov, K. Blaum, T. E. Cocolios, T. Day Goodacre, R. P. de Groote, A. de Roubin, G. J. Farooq-Smith, D. V. Fedorov, V. N. Fedosseev, R. Ferrer, D. A. Fink, L. P. Gaffney, L. Ghys et al., Phys. Lett. B 786, 355 (2018).
[16] M. Venhart, M. Balogh, A. Herzáň, J. L. Wood, F. A. Ali, D. T. Joss, A. N. Andreyev, K. Auranen, R. J. Carroll, M. C. Drummond, J. L. Easton, P. T. Greenlees, T. Grahn, A. Gredley, J. Henderson, U. Jakobsson, R. Julin, S. Juutinen, J. Konki, E. A. Lawrie et al., Phys. Lett. B 806, 135488 (2020)

[17] M. Sedlák, M. Venhart, J. L. Wood, V. Matoušek, M. Balogh, A. J. Boston, T. E. Cocolios, L. J. Harkness-Brennan, R.-D. Herzberg, D. T. Joss, D. S. Judson, J. Kliman, R. D. Page, A. Patel, K. Petrík, and M. Veselský, Eur. Phys. J. A 56, 161 (2020).

[18] A. E. Barzakh, D. Atanasov, A. N. Andreyev, M. Al Monthery, N. A. Althubiti, B. Andel, S. Antalic, K. Blaum, T. E. Cocolios, J. G. Cubiss, P. Van Duppen, T. D. Goodacre, A. de Roubin, Y. A. Demidov, G. J. Farooq-Smith, D. V. Fedorov, V. N. Fedosseev, D. A. Fink, L. P. Gaffney, L. Ghys et al., Phys. Rev. C 101, 034308 (2020).

[19] A. E. Barzakh, D. Atanasov, A. N. Andreyev, M. A. Monthery, N. A. Althubiti, B. Andel, S. Antalic, K. Blaum, T. E. Cocolios, J. G. Cubiss, P. Van Duppen, T. Day Goodacre, A. de Roubin, G. J. Farooq-Smith, D. V. Fedorov, V. N. Fedosseev, D. A. Fink, L. P. Gaffney, L. Ghys, R. D. Harding et al., Phys. Rev. C 101, 064321 (2020).

[20] R. Catherall, W. Andreazza, M. Breitenfeldt, A. Dorsival, G. J. Focker, T. P. Gharsa, T. J. Giles, J.-L. Grenard, F. Locci, P. Martins, S. Marzari, J. Schipper, A. Shornikov, and T. Stora, J. Phys. G: Nucl. Part. Phys. 44, 094002 (2017).

[21] R. D. Harding, A. N. Andreyev, A. E. Barzakh, D. Atanasov, J. G. Cubiss, P. Van Duppen, M. Al Monthery, N. A. Althubiti, B. Andel, S. Antalic, K. Blaum, T. E. Cocolios, T. Day Goodacre, A. de Roubin, G. J. Farooq-Smith, D. V. Fedorov, V. N. Fedosseev, D. A. Fink, L. P. Gaffney, L. Ghys et al., Phys. Rev. C 102, 024312 (2020).

[22] J. G. Cubiss, A. N. Andreyev, A. E. Barzakh, V. Manea, M. A. Monthery, N. A. Althubiti, B. Andel, S. Antalic, D. Atanasov, K. Blaum, T. E. Cocolios, T. D. Goodacre, A. de Roubin, G. J. Farooq-Smith, D. V. Fedorov, V. N. Fedosseev, D. A. Fink, L. P. Gaffney, L. Ghys, R. D. Harding et al., Phys. Rev. C 102, 044332 (2020).

[23] V. Fedosseev, K. Chrysalidis, T. Day Goodacre, B. Marsh, S. Rothe, C. Seiffert, and K. Wendt, J. Phys. G: Nucl. Part. Phys. 44, 084006 (2017).

[24] L. Ghys, A. N. Andreyev, M. Huyse, P. Van Duppen, S. Antalic, A. E. Barzakh, L. Capponi, T. E. Cocolios, J. Cubiss, X. Derkx, H. De Witte, J. Elseviers, F. P. Heßberger, Z. Kalaninová, U. Köster, J. F. W. Lane, V. Liberati, S. Mitsuoka, Y. Nagame, K. Nishio et al., Phys. Rev. C 100, 054310 (2019).

[25] A. Siivola, Nucl. Phys. A 109, 231 (1968).

[26] C. M. Baglin, Nucl. Data Sheets 110, 265 (2009).

[27] J. Keller, K.-H. Schmidt, F. P. Heßberger, G. Münzenberg, W. Reisdorf, H.-G. Clerc, and C.-C. Sahm, Nucl. Phys. A 452, 173 (1986).

[28] S. A. Gillespie, A. N. Andreyev, M. A. Monthery, C. J. Barton, S. Antalic, K. Auranen, H. Badran, D. Cox, J. G. Cubiss, D. O’Donnell, T. Grahn, P. T. Greenlees, A. Herzan, E. Higgins, R. Julin, S. Juutinen, J. Klimo, J. Konki, M. Leino, M. Mallaburn et al., Phys. Rev. C 99, 064310 (2019).

[29] J. O. Rasmussen, Phys. Rev. 113, 1593 (1959).

[30] Z. Kalaninová, S. Antalic, A. N. Andreyev, F. P. Heßberger, D. Ackermann, B. Andel, L. Bianco, S. Hofmann, M. Huyse, B. Kindler, B. Lommel, R. Mann, R. D. Page, P. J. Sapple, 
J. Thomson, P. Van Duppen, and M. Venhart, Phys. Rev. C 89, 054312 (2014).

[31] X. Huang and M. Kang, Nucl. Data Sheets 133, 221 (2016).

[32] A. Rytz, At. Data Nucl. Data Tables 47, 205 (1991).

[33] M. J. Oreglia, A Study of the Reactions $\psi^{\prime} \rightarrow \gamma \gamma \psi$, Ph.D. thesis, SLAC-R-236, 1980.

[34] P. M. Raddon, D. G. Jenkins, C. D. O’Leary, A. J. Simons, R. Wadsworth, A. N. Andreyev, R. D. Page, M. P. Carpenter, F. G. Kondev, T. Enqvist, P. T. Greenlees, P. M. Jones, R. Julin, S. Juutinen, H. Kettunen, M. Leino, A.-P. Leppänen, P. Nieminen, J. Pakarinen, P. Rahkila et al., Phys. Rev. C 70, 064308 (2004).

[35] T. Kibédi, T. W. Burrows, M. B. Trzhaskovskaya, P. M. Davidson, and C. W. Nestor, Nucl. Instrum. Methods A 589, 202 (2008)

[36] M. S. Basunia, Nucl. Data Sheets 102, 719 (2004).

[37] H. Gauvin, R. L. Hahn, Y. Le Beyec, M. Lefort, and J. Livet, Nucl. Phys. A 208, 360 (1973).

[38] F. G. Kondev, Nucl. Data Sheets 159, 1 (2019).

[39] C. M. Baglin, Nucl. Data Sheets 109, 2033 (2008).

[40] S. Juutinen, P. Ahonen, J. Hattula, R. Julin, A. Pakkanen, A. Virtanen, J. Simpson, R. Chapman, D. Clarke, F. Khazaie, J. Lisle, and J. N. Mo, Nucl. Phys. A 526, 346 (1991).

[41] M. Wang, G. Audi, F. G. Kondev, W. J. Huang, S. Naimi, and X. Xu, Chin. Phys. C 41, 030003 (2017).

[42] W. D. Schmidt-Ott, H. Salewski, F. Meissner, U. Bosch-Wicke, P. Koschel, V. Kunze, and R. Michaelsen, Nucl. Phys. A 545, 646 (1992).

[43] See Supplemental Material at http://link.aps.org/supplemental/ 10.1103/PhysRevC.104.024326 for determination of ${ }^{177} \mathrm{Au}^{\mathrm{m}}$ and ${ }^{177} \mathrm{Au}^{\mathrm{g}}$ alpha-decay branching ratios.

[44] J. Lettry, R. Catherall, P. Drumm, P. Van Duppen, A. H. M. Evensen, G. J. Focker, A. Jokinen, O. C. Jonsson, E. Kugler, H. Ravn et al. (ISOLDE Collaboration), Nucl. Instrum. Meth. Phys. Res. Sect. B: Beam Interact. Mater. Atoms 126, 130 (1997).

[45] C. Cabot, C. Deprun, H. Gauvin, B. Lagarde, Y. Le Beyec, and M. Lefort, Nucl. Phys. A 241, 341 (1975).

[46] P. M. Davidson, G. D. Dracoulis, T. Kibédi, A. P. Byrne, S. S. Anderssen, A. M. Baxter, B. Fabricius, G. J. Lane, and A. E. Stuchbery, Nucl. Phys. A 657, 219 (1999).

[47] B. Andel, A. N. Andreyev, S. Antalic, A. Barzakh, N. Bree, T. E. Cocolios, V. F. Comas, J. Diriken, J. Elseviers, D. V. Fedorov, V. N. Fedosseev, S. Franchoo, L. Ghys, J. A. Heredia, M. Huyse, O. Ivanov, U. Köster, V. Liberati, B. A. Marsh, K. Nishio et al., Phys. Rev. C 96, 054327 (2017).

[48] M. W. Rowe, J. C. Batchelder, T. N. Ginter, K. E. Gregorich, F. Q. Guo, F. P. Heßberger, V. Ninov, J. Powell, K. S. Toth, X. J. Xu, and J. Cerny, Phys. Rev. C 65, 054310 (2002).
[49] B. Singh, NNDC [www.nndc.bnl.gov], evaluated nuclear structure data file for ${ }^{172} \mathrm{Ir}$.

[50] S. Della Negra, C. Deprun, D. Jacquet, and Y. Le Beyec, Ann. Phys. 7, 149 (1982).

[51] F. G. Kondev, M. P. Carpenter, S. Zhu, R. Janssens, I. Ahmad, B. B. Back, P. F. Bertone, J. Chen, C. J. Chiara, C. A. Copos, J. P. Greene, C. R. Hoffman, B. P. Kay, T. L. Khoo, T. Lauritsen, E. A. McCutchan, C. Nair, A. M. Rogers, D. Seweryniak, and D. J. Hartley, EPJ Web Conf. 63, 01013 (2013).

[52] M. S. Basunia, Nucl. Data Sheets 107, 791 (2006).

[53] C. Ekström, L. Robertsson, S. Ingelman, G. Wannberg, and I. Ragnarsson, Nucl. Phys. A 348, 25 (1980).

[54] A. Bohr and V. F. Weisskopf, Phys. Rev. 77, 94 (1950).

[55] H. Dahmen and S. Penselin, Z. Phys. 200, 456 (1967).

[56] F. D. Feiock and W. R. Johnson, Phys. Rev. 187, 39 (1969).

[57] C. Ekström, G. Wannberg, and Y. S. Shishodia, Hyperfine Interact. 1, 437 (1975).

[58] A. N. Andreyev, A. Barzakh, B. Andel, S. Antalic, D. Atanasov, B. Bastin, K. Blaum, Ch. Borgmann, T. E. Cocolios, T. Day Goodacre et al.,CERN Proposal INTC-P-319-ADD-1, 2013.

[59] J. G. Cubiss, A. E. Barzakh, M. D. Seliverstov, A. N. Andreyev, B. Andel, S. Antalic, P. Ascher, D. Atanasov, D. Beck, J. Bieroń, K. Blaum, C. Borgmann, M. Breitenfeldt, L. Capponi, T. E. Cocolios, T. Day Goodacre, X. Derkx, H. De Witte, J. Elseviers, D. V. Fedorov et al., Phys. Rev. C 97, 054327 (2018).

[60] S. Sels, T. Day Goodacre, B. A. Marsh, A. Pastore, W. Ryssens, Y. Tsunoda, N. Althubiti, B. Andel, A. N. Andreyev, D. Atanasov, A. E. Barzakh, M. Bender, J. Billowes, K. Blaum, T. E. Cocolios, J. G. Cubiss, J. Dobaczewski, G. J. FarooqSmith, D. V. Fedorov, V. N. Fedosseev et al., Phys. Rev. C 99, 044306 (2019).

[61] A. E. Barzakh, A. N. Andreyev, T. E. Cocolios, R. P. de Groote, D. V. Fedorov, V. N. Fedosseev, R. Ferrer, D. A. Fink, L. Ghys, M. Huyse, U. Köster, J. Lane, V. Liberati, K. M. Lynch, B. A. Marsh, P. L. Molkanov, T. J. Procter, E. Rapisarda, S. Rothe, K. Sandhu et al., Phys. Rev. C 95, 014324 (2017).

[62] K. Van de Vel, A. N. Andreyev, M. Huyse, P. Van Duppen, J. F. C. Cocks, O. Dorvaux, P. T. Greenlees, K. Helariutta, P. Jones, R. Julin, S. Juutinen, H. Kettunen, P. Kuusiniemi, M. Leino, M. Muikku, P. Nieminen, K. Eskola, and R. Wyss, Phys. Rev. C 65, 064301 (2002).

[63] A. Bouldjedri, R. Duffait, R. Béraud, A. Emsallem, N. Redon, A. Gizon, J. Genevey, D. Barnéoud, and J. Blachot, Z. Phys. A: Hadrons Nucl. 342, 267 (1992).

Correction: An affiliation for the seventh author was improperly presented as a byline footnote and has been moved into the list of affiliations. Subsequent affiliations and footnotes have been reordered. 